\begin{tabular}{|c|c|c|c|}
\hline \multicolumn{4}{|c|}{35} \\
\hline $\mathrm{Zn}$ & 0.000051 & -0.000038 & 0.82577 \\
\hline $\mathrm{H}$ & 2.328025 & -0.491416 & -1.330918 \\
\hline $\mathrm{C}$ & 1.926028 & -1.445203 & \\
\hline $\mathrm{N}$ & & -1.4 & \\
\hline $\mathrm{C}$ & 1.915059 & -3.821398 & 192 \\
\hline $\mathrm{C}$ & & & \\
\hline $\mathrm{C}$ & 2.4 & -2.6 & -1. \\
\hline $\mathrm{C}$ & 0.8 & & \\
\hline $\mathrm{C}$ & -0.6 & -2. & \\
\hline $\mathrm{H}$ & & & \\
\hline $\mathrm{H}$ & 0.5 & -6.0 & \\
\hline $\mathrm{H}$ & 2.2 & -4 & -1 . \\
\hline $\mathrm{C}$ & -1.1 & & \\
\hline $\mathrm{H}$ & -1.9 & -3 . & \\
\hline $\mathrm{C}$ & -0.7 & & \\
\hline $\mathrm{H}$ & -1.2 & -5 & \\
\hline $\mathrm{C}$ & & $-5 .($ & \\
\hline $\mathrm{H}$ & -2.3 & & \\
\hline $\mathrm{C}$ & & & \\
\hline $\mathrm{N}$ & -0.9 & & \\
\hline $\mathrm{C}$ & -1.9 & & \\
\hline $\mathrm{C}$ & -0.4 & & \\
\hline $\mathrm{C}$ & -2.4 & & \\
\hline $\mathrm{C}$ & -0.8 & & -0 \\
\hline $\mathrm{C}$ & 0.64 & & \\
\hline $\mathrm{H}$ & & & 940 \\
\hline $\mathrm{H}$ & -0.5 & & 711 \\
\hline $\mathrm{H}$ & & & \\
\hline $\mathrm{C}$ & 1.19 & 3.7 & \\
\hline $\mathrm{H}$ & 1.97 & & 660 \\
\hline $\mathrm{C}$ & 0.741 & 4.9 & 427 \\
\hline $\mathrm{H}$ & 1.20 & & \\
\hline $\mathrm{C}$ & -0.260846 & 5.06 & 0.151072 \\
\hline $\mathrm{O}$ & & & \\
\hline $\mathrm{O}$ & 1 (1) 170? & -1.378579 & 16050 \\
\hline
\end{tabular}

\title{
Znq2 Dimer
}

70

scf done: -5422.757673

$\begin{array}{llll}\text { Zn } & 20.757354 & 26.210678 & 9.869104\end{array}$

$\begin{array}{llll}\mathrm{Zn} & 18.780768 & 27.795425 & 8.099317\end{array}$

$\begin{array}{llll}\text { O } & 18.719704 & 26.788963 & 9.745987\end{array}$

$\begin{array}{llll}\text { O } & 22.574480 & 25.519201 & 10.171987\end{array}$ 


$\begin{array}{lccc}\mathrm{O} & 20.817636 & 27.210055 & 8.216646 \\ \mathrm{O} & 16.965262 & 28.498243 & 7.807106 \\ \mathrm{~N} & 19.835443 & 24.431141 & 10.113658 \\ \mathrm{~N} & 21.173990 & 27.332858 & 11.506021 \\ \mathrm{~N} & 19.708162 & 29.573233 & 7.861570 \\ \mathrm{~N} & 18.354514 & 26.688982 & 6.454656 \\ \mathrm{C} & 20.462296 & 23.298442 & 10.284550 \\ \mathrm{C} & 19.761865 & 22.104478 & 10.542324 \\ \mathrm{C} & 18.405093 & 22.136410 & 10.627217 \\ \mathrm{C} & 16.300624 & 23.489827 & 10.520997 \\ \mathrm{C} & 15.741807 & 24.714622 & 10.321208 \\ \mathrm{C} & 16.526224 & 25.857014 & 10.058505 \\ \mathrm{C} & 17.886011 & 25.770053 & 10.004058 \\ \mathrm{C} & 18.479014 & 24.491131 & 10.196555 \\ \mathrm{C} & 17.706891 & 23.357580 & 10.454947 \\ \mathrm{C} & 20.415901 & 28.198722 & 12.121565 \\ \mathrm{C} & 20.881649 & 28.912758 & 13.240814 \\ \mathrm{C} & 22.146549 & 28.689425 & 13.688248 \\ \mathrm{C} & 24.302825 & 27.444493 & 13.439001 \\ \mathrm{C} & 25.000120 & 26.509522 & 12.732446 \\ \mathrm{C} & 24.450812 & 25.840193 & 11.625484 \\ \mathrm{C} & 23.172903 & 26.097648 & 11.192241 \\ \mathrm{C} & 22.440300 & 27.083366 & 11.936149 \\ \mathrm{C} & 22.982351 & 27.746894 & 13.038518 \\ \mathrm{C} & 19.084375 & 30.708781 & 7.697998 \\ \mathrm{C} & 19.787771 & 31.901579 & 7.443366 \\ \mathrm{C} & 21.144175 & 31.865601 & 7.353672 \\ \mathrm{C} & 23.244550 & 30.505007 & 7.446225 \\ \mathrm{C} & 23.800097 & 29.277656 & 7.638746 \\ \mathrm{C} & 23.012884 & 28.136635 & 7.899464 \\ \mathrm{C} & 21.653639 & 28.227530 & 7.959104 \\ \mathrm{C} & 21.064042 & 29.509206 & 7.773958 \\ \mathrm{C} & 21.838905 & 30.641433 & 7.517868 \\ \mathrm{C} & 19.106648 & 25.823652 & 5.831086 \\ \mathrm{C} & 18.636046 & 25.123250 & 4.705308 \\ \mathrm{C} & 17.372799 & 25.359641 & 4.259882 \\ \mathrm{C} & 15.225352 & 26.617372 & 4.520290 \\ \mathrm{C} & 14.534322 & 27.550333 & 5.235587 \\ \mathrm{C} & 15.087841 & 28.205040 & 6.349170 \\ \mathrm{C} & 16.363651 & 27.934324 & 6.780577 \\ \mathrm{C} & 17.089859 & 26.951042 & 6.027110 \\ \mathrm{C} & 16.543553 & 26.301961 & 4.918232 \\ \mathrm{H} & 21.529082 & 23.337172 & 10.231113 \\ \mathrm{H} & 20.306590 & 21.193315 & 10.673616 \\ \mathrm{H} & 17.847712 & 21.242058 & 10.826418 \\ \mathrm{H} & 15.696385 & 22.627133 & 10.719006\end{array}$




$\begin{array}{cccc}\mathrm{H} & 14.676128 & 24.824845 & 10.357024 \\ \mathrm{H} & 16.063171 & 26.803796 & 9.874368 \\ \mathrm{H} & 19.428386 & 28.333649 & 11.733799 \\ \mathrm{H} & 20.236877 & 29.617576 & 13.722522 \\ \mathrm{H} & 22.526871 & 29.220932 & 14.538700 \\ \mathrm{H} & 24.733014 & 27.946455 & 14.282324 \\ \mathrm{H} & 26.003849 & 26.269245 & 13.025646 \\ \mathrm{H} & 25.031747 & 25.111571 & 11.099151 \\ \mathrm{H} & 18.017688 & 30.673063 & 7.754997 \\ \mathrm{H} & 19.245655 & 32.815172 & 7.318311 \\ \mathrm{H} & 21.703769 & 32.759077 & 7.156752 \\ \mathrm{H} & 23.850790 & 31.366756 & 7.250230 \\ \mathrm{H} & 24.865267 & 29.164064 & 7.598714 \\ \mathrm{H} & 23.473938 & 27.187951 & 8.078585 \\ \mathrm{H} & 20.093389 & 25.678725 & 6.217263 \\ \mathrm{H} & 19.275849 & 24.418334 & 4.217167 \\ \mathrm{H} & 16.989025 & 24.838981 & 3.404286 \\ \mathrm{H} & 14.791925 & 26.126381 & 3.672181 \\ \mathrm{H} & 13.532437 & 27.800498 & 4.944359 \\ \mathrm{H} & 14.511651 & 28.932296 & 6.882569\end{array}$

\section{Znq2 Trimer}

\begin{tabular}{|c|c|c|c|}
\hline & e. -01 & & \\
\hline $\mathrm{Zn}$ & -2.670268 & -0.797332 & -0.299969 \\
\hline $\mathrm{O}$ & -4.651310 & -0.666145 & -0.263332 \\
\hline $\mathrm{O}$ & -0.628312 & -1.309554 & -0.459816 \\
\hline $\mathrm{N}$ & -2.899768 & -0.824550 & 1.721939 \\
\hline $\mathrm{N}$ & -2.772177 & -2.470190 & -1.457182 \\
\hline $\mathrm{C}$ & -1.984141 & -0.847980 & 2.652838 \\
\hline $\mathrm{C}$ & -2.324448 & -0.913773 & 4.016888 \\
\hline $\mathrm{C}$ & -3.635943 & -0.944567 & 4.371875 \\
\hline $\mathrm{C}$ & -6.032216 & -0.891942 & 3.659606 \\
\hline $\mathrm{C}$ & -6.908261 & -0.816134 & 2.617564 \\
\hline $\mathrm{C}$ & -6.478715 & -0.737245 & 1.282266 \\
\hline $\mathrm{C}$ & -5.144135 & -0.738262 & 0.955238 \\
\hline $\mathrm{C}$ & -4.222622 & -0.825731 & 2.051658 \\
\hline $\mathrm{C}$ & -4.647489 & -0.892629 & 3.380207 \\
\hline C & -3.880855 & -2.989249 & -1.913460 \\
\hline $\mathrm{C}$ & -3.873951 & -4.151632 & -2.708733 \\
\hline $\mathrm{C}$ & -2.688426 & -4.746084 & -3.006273 \\
\hline $\mathrm{C}$ & -0.195464 & -4.738028 & -2.787127 \\
\hline $\mathrm{C}$ & 0.900474 & -4.112638 & -2.277094 \\
\hline C & 0.795001 & -2.951209 & -1.486052 \\
\hline
\end{tabular}




\begin{tabular}{|c|c|c|c|}
\hline $\mathrm{C}$ & 742 & 941 & \\
\hline $\mathrm{C}$ & & -3.046565 & \\
\hline $\mathrm{C}$ & & & \\
\hline n & 0.051248 & & \\
\hline $\mathrm{Zn}$ & & & \\
\hline 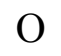 & 2.10 & 0.4 & \\
\hline $\mathrm{O}$ & -1.9 & & \\
\hline 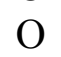 & 0.5 & & \\
\hline $\mathrm{O}$ & & & \\
\hline $\mathrm{N}$ & 0.3 & & \\
\hline $\mathrm{N}$ & -0.1 & & \\
\hline $\mathrm{N}$ & 81 & 2.0 & \\
\hline $\mathrm{N}$ & 2.6 & -1. & \\
\hline $\mathrm{C}$ & -0.5 & & \\
\hline $\mathrm{C}$ & -0.1 & -0. & \\
\hline C & & & \\
\hline 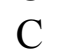 & 3.5 & -0 . & \\
\hline 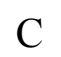 & & & \\
\hline f & 3.9 & & -2 \\
\hline 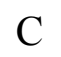 & & & \\
\hline $\mathrm{C}$ & 1.6 & & \\
\hline $\mathrm{C}$ & & & \\
\hline $\mathrm{C}$ & & & \\
\hline $\mathrm{C}$ & 0.6 & & \\
\hline $\mathrm{C}$ & & & \\
\hline $\mathrm{C}$ & -3.0 & & \\
\hline $\mathrm{C}$ & -4.0 & & \\
\hline $\mathrm{C}$ & -3.7 & & -1 . \\
\hline $\mathrm{C}$ & -2.4 & & \\
\hline $\mathrm{C}$ & -1.3 & & -1 . \\
\hline $\mathrm{C}$ & -1.7 & & \\
\hline $\mathrm{C}$ & & & \\
\hline 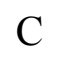 & 3.8 & & \\
\hline $\mathrm{C}$ & 2.6 & 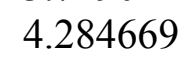 & \\
\hline 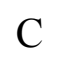 & 0.1 & 4.2 & \\
\hline $\mathrm{C}$ & -0.9 & 9 & \\
\hline S & -0.8 & & \\
\hline $\mathrm{C}$ & 0.3 & & \\
\hline $\mathrm{C}$ & 1.5 & 2.5 & \\
\hline $\mathrm{C}$ & 1.4 & & \\
\hline $\mathrm{C}$ & 1.6 & -2.3 & \\
\hline 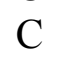 & & -3.5 & \\
\hline $\mathrm{C}$ & 3.1 & -3.9 & 2.6 \\
\hline $\mathrm{C}$ & 5.6 & -3. & \\
\hline $\mathrm{C}$ & 6.594441 & -2.5 & 2.24 \\
\hline 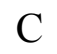 & 6.287035 & -1.340939 & 1.6817 \\
\hline
\end{tabular}




$\begin{array}{lrrr}\mathrm{C} & 4.987903 & -0.953265 & 1.458016 \\ \mathrm{C} & 3.971745 & -1.894411 & 1.833013 \\ \mathrm{C} & 4.275809 & -3.141655 & 2.382600 \\ \mathrm{H} & -0.967726 & -0.778014 & 2.333052 \\ \mathrm{H} & -1.546787 & -0.929480 & 4.751315 \\ \mathrm{H} & -3.922715 & -0.995951 & 5.404129 \\ \mathrm{H} & -6.370950 & -0.945603 & 4.674833 \\ \mathrm{H} & -7.962839 & -0.811530 & 2.814981 \\ \mathrm{H} & -7.197763 & -0.668106 & 0.492464 \\ \mathrm{H} & -4.780913 & -2.481020 & -1.645776 \\ \mathrm{H} & -4.801000 & -4.549262 & -3.065093 \\ \mathrm{H} & -2.655110 & -5.632173 & -3.609718 \\ \mathrm{H} & -0.106079 & -5.621631 & -3.386980 \\ \mathrm{H} & 1.878088 & -4.504720 & -2.477017 \\ \mathrm{H} & 1.680416 & -2.483375 & -1.110811 \\ \mathrm{H} & -1.553991 & 0.206190 & -3.529437 \\ \mathrm{H} & -0.969348 & -0.098750 & -5.929917 \\ \mathrm{H} & 1.406390 & -0.228433 & -6.573561 \\ \mathrm{H} & 3.856441 & -0.198826 & -5.825381 \\ \mathrm{H} & 5.443698 & -0.025693 & -3.967149 \\ \mathrm{H} & 4.652642 & 0.279363 & -1.644636 \\ \mathrm{H} & 1.861623 & 3.143307 & -1.250634 \\ \mathrm{H} & 1.469012 & 5.532919 & -1.827769 \\ \mathrm{H} & -0.845633 & 6.323981 & -2.130079 \\ \mathrm{H} & -3.344800 & 5.760449 & -2.117882 \\ \mathrm{H} & -5.073143 & 4.055207 & -1.789942 \\ \mathrm{H} & -4.468563 & 1.726107 & -1.222181 \\ \mathrm{H} & 4.747025 & 2.103802 & 2.181065 \\ \mathrm{H} & 4.766185 & 4.142529 & 3.642367 \\ \mathrm{H} & 2.614627 & 5.156380 & 4.288294 \\ \mathrm{H} & 0.061216 & 5.100392 & 4.133575 \\ \mathrm{H} & -1.923925 & 3.985991 & 3.222498 \\ \mathrm{H} & -1.724719 & 1.999720 & 1.805991 \\ \mathrm{H} & 0.713299 & -1.977724 & 1.605391 \\ \mathrm{H} & 1.071830 & -4.225670 & 2.617411 \\ & 3.374493 & -4.958060 & 3.104098 \\ \mathrm{H} & 7.875065 & -4.440466 & 3.018717 \\ & 7.074291 & -0.668531 & 1.410306\end{array}$




\section{Znq2 Tetramer}

$$
\begin{array}{lrrr}
140 & & & \\
\mathrm{ZN} & -1.2882993731 & -0.6007530831 & 0.6526690381 \\
\mathrm{ZN} & -4.3720950420 & -0.5272155961 & 0.1206973904 \\
\mathrm{O} & -3.1267117004 & -0.2679942797 & 1.5454720623 \\
\mathrm{O} & 0.5794907376 & -1.1218547318 & 0.0067759306 \\
\mathrm{O} & -2.5889497384 & -1.0696945655 & -0.8582754181 \\
\mathrm{O} & -6.1609490785 & -0.2375758967 & 0.9458871982 \\
\mathrm{~N} & -0.7844759808 & -0.1976508488 & 2.7213641144 \\
\mathrm{~N} & -1.1285100040 & -2.7008374083 & 1.2369493215 \\
\mathrm{~N} & -4.8746138821 & -2.3712483355 & -0.5597228272 \\
\mathrm{~N} & -4.9401970167 & 1.0572960515 & -1.0229410449 \\
\mathrm{C} & 0.4112473726 & -0.1694033841 & 3.2392365378 \\
\mathrm{C} & 0.6144420326 & 0.0041220091 & 4.6249080618 \\
\mathrm{C} & -0.4617190029 & 0.1530519719 & 5.4402084746 \\
\mathrm{C} & -2.9528325846 & 0.2992708457 & 5.6580929543 \\
\mathrm{C} & -4.1567392332 & 0.2739683094 & 5.0232433965 \\
\mathrm{C} & -4.2614832051 & 0.0895335761 & 3.6292091402 \\
\mathrm{C} & -3.1407968329 & -0.0695532817 & 2.8668989875 \\
\mathrm{C} & -1.8760915013 & -0.0403865152 & 3.5157041708 \\
\mathrm{C} & -1.7710138968 & 0.1394303371 & 4.8970591874 \\
\mathrm{C} & -2.0059853046 & -3.4256698718 & 1.8727559752 \\
\mathrm{C} & -1.7807139500 & -4.7881472461 & 2.1554783344 \\
\mathrm{C} & -0.6236094999 & -5.3670665509 & 1.7416906748 \\
\mathrm{C} & 1.5664009933 & -5.1199478436 & 0.5567677207 \\
\mathrm{C} & 2.4104923675 & -4.2924476986 & -0.1180920069 \\
\mathrm{C} & 2.1080421061 & -2.9328109955 & -0.3320246316 \\
\mathrm{C} & 0.9446207697 & -2.3902879501 & 0.1384814780 \\
\mathrm{C} & 0.0413688924 & -3.2551743550 & 0.8247295460 \\
\mathrm{C} & 0.3427038255 & -4.6018433701 & 1.0431640465 \\
\mathrm{C} & -6.0278359038 & -2.9512057683 & -0.3605077198 \\
\mathrm{C} & -6.3199881481 & -4.2108859655 & -0.9170499401 \\
\mathrm{C} & -5.3821500570 & -4.8306162271 & -1.6817151443 \\
\mathrm{C} & -3.0891970022 & -4.7914196274 & -2.6793438857 \\
\mathrm{C} & -1.9167750414 & -4.1134553224 & -2.8101257626 \\
\mathrm{C} & -1.7123505876 & -2.8545954756 & -2.2110529618 \\
\mathrm{C} & -2.6923576246 & -2.2556125228 & -1.4706017385 \\
\mathrm{C} & -3.9184190891 & -2.9679585493 & -1.3190086508 \\
\mathrm{C} & -4.1260354489 & -4.2163162987 & -1.9103994145 \\
\mathrm{C} & -4.2940365835 & 1.6411507999 & -1.9937498869 \\
\mathrm{C} & -6.06665938917 & 2.7363555265 & -2.6899181776 \\
\mathrm{C} & -8.0708155874 & 2.9929549692 & -0.8593717989 \\
\hline
\end{array}
$$




$\begin{array}{lccc}\mathrm{C} & -6.8060718566 & 0.7813719988 & 0.4188561228 \\ \mathrm{C} & -6.1770686316 & 1.4972085609 & -0.6535346634 \\ \mathrm{C} & -6.7899986374 & 2.5800522029 & -1.2880062360 \\ \mathrm{ZN} & 1.2882657254 & 0.6007118784 & -0.6527370627 \\ \mathrm{ZN} & 4.3722939768 & 0.5280879517 & -0.1203869079 \\ \mathrm{O} & 3.1269665440 & 0.2686410374 & -1.5452692664 \\ \mathrm{O} & -0.5794895027 & 1.1219424277 & -0.0064298113 \\ \mathrm{O} & 2.5888849266 & 1.0695251997 & 0.8584367996 \\ \mathrm{O} & 6.1611651187 & 0.2395366132 & -0.9462630976 \\ \mathrm{~N} & 0.7847326530 & 0.1966540079 & -2.7212029184 \\ \mathrm{~N} & 1.1284571481 & 2.7006683242 & -1.2369473169 \\ \mathrm{~N} & 4.8739943729 & 2.3720578326 & 0.5606987613 \\ \mathrm{~N} & 4.9413344058 & -1.0565217444 & 1.0224485087 \\ \mathrm{C} & -0.4109312597 & 0.1674424753 & -3.2391050191 \\ \mathrm{C} & -0.6139864639 & -0.0064816398 & -4.6247860087 \\ \mathrm{C} & 0.4622791737 & -0.1547371741 & -5.4400274728 \\ \mathrm{C} & 2.9534744707 & -0.2993615235 & -5.6578070341 \\ \mathrm{C} & 4.1573775308 & -0.2731862242 & -5.0229366646 \\ \mathrm{C} & 4.2619733877 & -0.0885429558 & -3.6289583269 \\ \mathrm{C} & 3.1411581039 & 0.0699712390 & -2.8666672900 \\ \mathrm{C} & 1.8765109855 & 0.0399544747 & -3.5154981092 \\ \mathrm{C} & 1.7715745393 & -0.1401539181 & -4.8968210229 \\ \mathrm{C} & 2.0059838090 & 3.4254019183 & -1.8728016556 \\ \mathrm{C} & 1.7807926840 & 4.7878743366 & -2.1556201041 \\ \mathrm{C} & 0.6237271295 & 5.3668944548 & -1.7418571490 \\ \mathrm{C} & -1.5662479138 & 5.1200210165 & -0.5568212568 \\ \mathrm{C} & -2.4103265149 & 4.2926367069 & 0.1182015524 \\ \mathrm{C} & -2.1079603558 & 2.9330083413 & 0.3322189700 \\ \mathrm{C} & -0.9446199355 & 2.3903489900 & -0.1383409062 \\ \mathrm{C} & -0.0413825560 & 3.2551123198 & -0.8247501168 \\ \mathrm{C} & -0.3426214318 & 4.6017902756 & -1.0432592216 \\ \mathrm{C} & 6.0269520263 & 2.9526108793 & 0.3616669917 \\ \mathrm{C} & 8.6679304267 & -2.3132920425 & -0.1653167791 \\ \mathrm{C} & 6.3184809757 & 4.2122882243 & 0.9185444109 \\ \mathrm{C} & 5.3803213415 & 4.8313683680 & 1.6833475830 \\ \mathrm{C} & 3.0873165467 & 4.7908190945 & 2.6808579725 \\ \mathrm{C} & 1.9151864180 & 4.1122948603 & 2.8113085267 \\ \mathrm{C} & 1.7113565065 & 2.8535210333 & 2.2118243574 \\ \mathrm{C} & 2.6916881552 & 2.2552147551 & 1.4712671472 \\ \mathrm{C} & 3.9174685627 & 2.9681585584 & 1.3200359689 \\ \mathrm{C} & 4.1244821995 & 4.2164237238 & 1.9118017527 \\ \mathrm{C} & 4.2955644303 & -1.6410417008 & 1.9931174583 \\ \mathrm{C} & 6.0687555371 & -3.1971498067 & 2.3373998464 \\ \mathrm{C} & -1.0724829198 & -2.9911772389 & 0.8572061853 \\ \mathrm{C} & -1.2218351597 & -0.7987029608\end{array}$




$\begin{array}{lrrr}\mathrm{C} & 6.8067256516 & -0.7794230063 & -0.4197692695 \\ \mathrm{C} & 6.1782297461 & -1.4959107139 & 0.6524909077 \\ \mathrm{C} & 6.7916477065 & -2.5788508401 & 1.2863346577 \\ \mathrm{H} & 1.2337869436 & -0.2492210918 & 2.5609753473 \\ \mathrm{H} & 1.6123106931 & 0.0293549897 & 5.0105327359 \\ \mathrm{H} & -0.3366094272 & 0.2899741044 & 6.4966488118 \\ \mathrm{H} & -2.8843045936 & 0.4396558047 & 6.7186362737 \\ \mathrm{H} & -5.0589938210 & 0.3963762053 & 5.5898134888 \\ \mathrm{H} & -5.2150595818 & 0.0681103281 & 3.1442171482 \\ \mathrm{H} & -2.9144664086 & -2.9466514462 & 2.1726292989 \\ \mathrm{H} & -2.5256319008 & -5.3466665657 & 2.6829151247 \\ \mathrm{H} & -0.4287021175 & -6.4042693866 & 1.9329914394 \\ \mathrm{H} & 1.8061554976 & -6.1521995048 & 0.7177504600 \\ \mathrm{H} & 3.3380374548 & -4.6727591096 & -0.4989741221 \\ \mathrm{H} & 2.7924056788 & -2.3057990403 & -0.8632473263 \\ \mathrm{H} & -6.7280631362 & -2.4116887685 & 0.2393511413 \\ \mathrm{H} & -7.2741817919 & -4.6586370756 & -0.7339668746 \\ \mathrm{H} & -5.5788962813 & -5.7894978639 & -2.1202293954 \\ \mathrm{H} & -3.2360708987 & -5.7491135252 & -3.1374289524 \\ \mathrm{H} & -1.1148634311 & -4.5410888139 & -3.3788631412 \\ \mathrm{H} & -0.7690102913 & -2.3656820644 & -2.3193695348 \\ \mathrm{H} & -3.3254806825 & 1.2606774426 & -2.2308854539 \\ \mathrm{H} & -4.2727406505 & 3.1934847578 & -3.4746213430 \\ \mathrm{H} & -6.5041489023 & 4.0332667234 & -2.8497860229 \\ \mathrm{H} & -8.5508530359 & 3.8230849321 & -1.3376713508 \\ \mathrm{H} & -9.6391565446 & 2.6190236219 & 0.5002005013 \\ \mathrm{H} & -8.5513813385 & 0.7171232860 & 1.5958900839 \\ \mathrm{H} & -1.2336084107 & 0.2467585232 & -2.5609434907 \\ \mathrm{H} & -1.6118613492 & -0.0324417682 & -5.0103458894 \\ \mathrm{H} & 0.3373288363 & -0.2918612099 & -6.4964607118 \\ \mathrm{H} & 2.8850902943 & -0.4399542514 & -6.7183295379 \\ \mathrm{H} & 5.0596884071 & -0.3950526733 & -5.5895349092 \\ \mathrm{H} & 5.2154899613 & -0.0664374777 & -3.1438846846 \\ \mathrm{H} & 2.9144366117 & 2.9463200032 & -2.1726429329 \\ \mathrm{H} & 2.5257527254 & 5.3463208532 & -2.6830708693 \\ \mathrm{H} & 0.4288936583 & 6.4040924168 & -1.9332612220 \\ \mathrm{H} & -1.8059291363 & 6.1522761144 & -0.7178874304 \\ \mathrm{H} & -3.3378171232 & 4.6730658650 & 0.4991022545 \\ & -2.7922756490 & 2.3060872693 & 0.8636049712 \\ \mathrm{H} & 6.7274590456 & 2.4136087489 & -0.2383347141 \\ \mathrm{H} & 1.2724446274 & 4.6605729986 & 0.7355699652 \\ & & & \end{array}$


$\begin{array}{cccc}\mathrm{H} & 4.2751259559 & -3.1940509618 & 3.4733015419 \\ \mathrm{H} & 6.5066229607 & -4.0328478876 & 2.8475353405 \\ \mathrm{H} & 8.5529281999 & -3.8213130434 & 1.3350844892 \\ \mathrm{H} & 9.6403475011 & -2.6161864952 & -0.5026362156 \\ \mathrm{H} & 8.5517527176 & -0.7141556082 & -1.5971931971\end{array}$

\section{Meq2Zn monomer}

41

scf done: -2788.994638

$\begin{array}{crrc}\mathrm{Zn} & 0.032185 & -0.003423 & 0.989319 \\ \mathrm{C} & -2.085069 & 1.434157 & -0.638021 \\ \mathrm{~N} & -1.002383 & 1.467956 & 0.092724 \\ \mathrm{C} & -2.042118 & 3.820012 & -0.873168 \\ \mathrm{C} & -0.393108 & 2.654117 & 0.405641 \\ \mathrm{C} & -2.635925 & 2.631438 & -1.149309 \\ \mathrm{C} & -0.879140 & 3.872438 & -0.063168 \\ \mathrm{C} & 0.754889 & 2.568640 & 1.257092 \\ \mathrm{H} & -3.521273 & 2.580185 & -1.747596 \\ \mathrm{H} & -2.451269 & 4.734824 & -1.255475 \\ \mathrm{C} & 1.365492 & 3.751475 & 1.584202 \\ \mathrm{H} & 2.221424 & 3.722169 & 2.225100 \\ \mathrm{C} & 0.883721 & 4.981512 & 1.103651 \\ \mathrm{H} & 1.397926 & 5.878011 & 1.389956 \\ \mathrm{C} & -0.212597 & 5.063618 & 0.297035 \\ \mathrm{C} & 2.040265 & -1.424114 & -0.784312 \\ \mathrm{~N} & 1.006214 & -1.465256 & 0.013427 \\ \mathrm{C} & 1.982518 & -3.807745 & -1.038171 \\ \mathrm{C} & 0.418301 & -2.654686 & 0.353840 \\ \mathrm{C} & 2.557467 & -2.616334 & -1.340626 \\ \mathrm{C} & 0.873564 & -3.868355 & -0.156160 \\ \mathrm{C} & -0.672634 & -2.577882 & 1.278015 \\ \mathrm{H} & 3.402991 & -2.559142 & -1.993489 \\ \mathrm{H} & 2.366612 & -4.718761 & -1.454071 \\ \mathrm{C} & -1.260916 & -3.764127 & 1.632611 \\ \mathrm{H} & -2.074080 & -3.741278 & 2.327221 \\ \mathrm{C} & -0.810870 & -4.989325 & 1.110833 \\ \mathrm{H} & -1.305626 & -5.888807 & 1.421056 \\ \mathrm{C} & 0.231563 & -5.063239 & 0.234927 \\ \mathrm{O} & -1.027559 & -1.379772 & 1.719643 \\ \mathrm{O} & 1.136629 & 1.366379 & 1.663649 \\ \mathrm{H} & 0.572378 & -6.002514 & -0.152143 \\ \mathrm{H} & -0.576801 & 6.006489 & -0.058734 \\ \mathrm{C} & 2.654324 & -0.081297 & -1.093100 \\ \mathrm{H} & 2.525752 & 0.579485 & -0.246744\end{array}$




$\begin{array}{rrrr}\mathrm{H} & 2.165114 & 0.361513 & -1.956084 \\ \mathrm{H} & 3.708819 & -0.178921 & -1.314252 \\ \mathrm{C} & -2.718199 & 0.094063 & -0.919381 \\ \mathrm{H} & -2.283227 & -0.341722 & -1.814405 \\ \mathrm{H} & -3.784219 & 0.193782 & -1.074319 \\ \mathrm{H} & -2.537842 & -0.573729 & -0.088091\end{array}$

\section{Meq2Zn Dimer}

82

scf done: -5578.046788

$\begin{array}{llll}\mathrm{Zn} & 1.437832 & -0.578118 & 0.258538\end{array}$

$\begin{array}{llll}\mathrm{Zn} & -1.436544 & 0.581100 & -0.260417\end{array}$

$\begin{array}{llll}\mathrm{O} & -0.276435 & -0.939885 & -0.725049\end{array}$

$\begin{array}{llll}\mathrm{O} & 2.855954 & -0.113013 & 1.527018\end{array}$

$\begin{array}{llll}\mathrm{O} & 0.278329 & 0.943359 & 0.721088\end{array}$

$\begin{array}{llll}\mathrm{O} & -2.853944 & 0.116253 & -1.529911\end{array}$

$\begin{array}{llll}\mathrm{N} & 1.224300 & -2.670468 & 0.594804\end{array}$

$\begin{array}{llll}\mathrm{N} & 2.913833 & -0.160632 & -1.149616\end{array}$

$\begin{array}{llll}\mathrm{N} & -1.226893 & 2.674961 & -0.592243\end{array}$

$\begin{array}{llll}\mathrm{N} & -2.911893 & 0.159389 & 1.146555\end{array}$

$\begin{array}{llll}\text { C } & 1.933274 & -3.498355 & 1.323552\end{array}$

$\begin{array}{llll}\text { C } & 1.758014 & -4.904107 & 1.211548\end{array}$

$\begin{array}{llll}\text { C } & 0.854599 & -5.416148 & 0.349202\end{array}$

$\begin{array}{llll}\text { C } & -0.933701 & -4.990957 & -1.331913\end{array}$

$\begin{array}{llll}\text { C } & -1.676571 & -4.074110 & -2.011307\end{array}$

$\begin{array}{llll}\text { C } & -1.479709 & -2.694995 & -1.828869\end{array}$

$\begin{array}{llll}\text { C } & -0.523887 & -2.233183 & -0.969303\end{array}$

$\begin{array}{llll}\text { C } & 0.266839 & -3.174492 & -0.261995\end{array}$

C $\quad 0.054826 \quad-4.543431 \quad-0.430623$

$\begin{array}{llll}\text { C } & 2.892494 & -0.176097 & -2.458767\end{array}$

$\begin{array}{llll}\text { C } & 4.055737 & 0.137777 & -3.205296\end{array}$

$\begin{array}{llll}\text { C } & 5.205897 & 0.457912 & -2.570849\end{array}$

$\begin{array}{llll}\text { C } & 6.408195 & 0.820467 & -0.419094\end{array}$

$\begin{array}{llll}\text { C } & 6.341489 & 0.826429 & 0.942346\end{array}$

$\begin{array}{llll}\text { C } & 5.154852 & 0.514194 & 1.621179\end{array}$

$\begin{array}{llll}\text { C } & 4.002850 & 0.187803 & 0.947459\end{array}$

$\begin{array}{llll}\text { C } & 4.070053 & 0.173575 & -0.480920\end{array}$

$\begin{array}{llll}\text { C } & 5.250572 & 0.488707 & -1.154161\end{array}$

$\begin{array}{llll}\text { C } & -1.937990 & 3.503480 & -1.318091\end{array}$

$\begin{array}{llll}\text { C } & -1.764395 & 4.909284 & -1.203236\end{array}$

$\begin{array}{llll}\text { C } & -0.860448 & 5.420517 & -0.341023\end{array}$

$\begin{array}{llll}\text { C } & 0.930743 & 4.993870 & 1.336673\end{array}$

$\begin{array}{llll}\text { C } & 1.675916 & 4.076460 & 2.012856\end{array}$

$\begin{array}{llll}\text { C } & 1.480729 & 2.697525 & 1.827428\end{array}$ 


$\begin{array}{lrrr}\mathrm{C} & 0.524202 & 2.236398 & 0.968224 \\ \mathrm{C} & -0.268863 & 3.178257 & 0.264363 \\ \mathrm{C} & -0.058412 & 4.547075 & 0.435769 \\ \mathrm{C} & -2.890224 & 0.172518 & 2.455751 \\ \mathrm{C} & -4.052463 & -0.145546 & 3.201953 \\ \mathrm{C} & -5.201975 & -0.467427 & 2.567104 \\ \mathrm{C} & -6.403898 & -0.828904 & 0.414866 \\ \mathrm{C} & -6.337348 & -0.832155 & -0.946536 \\ \mathrm{C} & -5.151492 & -0.515987 & -1.625087 \\ \mathrm{C} & -4.000176 & -0.188124 & -0.951026 \\ \mathrm{C} & -4.067296 & -0.176539 & 0.477511 \\ \mathrm{C} & -5.246861 & -0.495743 & 1.150404 \\ \mathrm{H} & 2.364557 & -5.538910 & 1.823332 \\ \mathrm{H} & 0.723261 & -6.476033 & 0.250376 \\ \mathrm{H} & -1.090397 & -6.043570 & -1.461032 \\ \mathrm{H} & -2.436973 & -4.400079 & -2.693085 \\ \mathrm{H} & -2.090936 & -1.978205 & -2.331586 \\ \mathrm{H} & 3.998973 & 0.113781 & -4.273602 \\ \mathrm{H} & 6.092445 & 0.694977 & -3.126129 \\ \mathrm{H} & 7.314415 & 1.062257 & -0.937663 \\ \mathrm{H} & 7.212783 & 1.076498 & 1.515925 \\ \mathrm{H} & 5.127797 & 0.530996 & 2.691134 \\ \mathrm{H} & -2.372738 & 5.544582 & -1.812701 \\ \mathrm{H} & -0.730165 & 6.480336 & -0.239996 \\ \mathrm{H} & 1.086336 & 6.046384 & 1.467910 \\ \mathrm{H} & 2.436819 & 4.401872 & 2.694323 \\ \mathrm{H} & 2.093658 & 1.980298 & 2.327466 \\ \mathrm{H} & -3.995539 & -0.123367 & 4.270284 \\ \mathrm{H} & -6.087821 & -0.707748 & 3.122096 \\ \mathrm{H} & -7.309385 & -1.073855 & 0.933229 \\ \mathrm{H} & -7.208059 & -1.083207 & -1.520584 \\ \mathrm{H} & -5.124795 & -0.530403 & -2.695086 \\ \mathrm{C} & -1.630441 & 0.542953 & 3.196620 \\ \mathrm{H} & -0.771646 & 0.457032 & 2.554139 \\ \mathrm{H} & -1.503872 & -0.094665 & 4.063173 \\ \mathrm{H} & -1.696508 & 1.571317 & 3.540912 \\ \mathrm{H} & 1.631985 & -0.544192 & -3.199649 \\ \mathrm{H} & 1.696670 & -1.572346 & -3.544830 \\ \mathrm{H} & -2.773545 & -0.457738 & -2.556795 \\ \mathrm{H} & -2.752596 & 3.422052 & -3.283444 \\ \mathrm{H} & -3.344587 & -2.003304 \\ \mathrm{H} & -3.031288 & -2.346272 \\ \mathrm{H} & -3.410977 & 3.291092\end{array}$




$$
\begin{array}{llll}
\mathrm{H} & 2.959674 & -1.925308 & 2.348157 \\
\mathrm{H} & 3.946201 & -3.344149 & 2.014935
\end{array}
$$

\section{Meq2Zn trimer}

123

ZN $0.0189160494 \quad 0.3442246969 \quad 1.1024261446$

ZN $2.5660946229 \quad 0.8339487745 \quad-0.6949754554$

$\begin{array}{llll}\text { O } & 2.0375902754 & -0.0031207032 & 0.9956744343\end{array}$

$\begin{array}{llll}\text { O } & -2.0061346800 & 0.5959876656 & 0.8955000165\end{array}$

$\begin{array}{lllll}\mathrm{O} & 0.6364222173 & 1.3928055115 & -0.5148414858\end{array}$

$\begin{array}{lllll}\text { O } & 4.3851560161 & 0.1020940125 & -0.6947388317\end{array}$

N $0.4728081269 \quad-0.5620194097 \quad 3.0709345921$

$\mathrm{N}-0.3685870160 \quad 2.2103089144 \quad 2.2299699392$

N $2.8121433799 \quad 2.9107159009 \quad-0.7742527375$

N $2.5729876371 \quad-0.0570511941 \quad-2.6298885704$

C $-0.3374911918-0.8377498228 \quad 4.0595031393$

C $\quad 0.1444770071 \quad-1.4099972107 \quad 5.2641414403$

C $1.4598164358-1.6894602342 \quad 5.4015621866$

C $3.7362215540 \quad-1.6947663586 \quad 4.3955217678$

C $4.5114224295-1.4062004317 \quad 3.3157520453$

C $3.9673434238 \quad-0.8399143207 \quad 2.1493571986$

C $2.6322963148 \quad-0.55569282092 .0598656066$

C $1.8080061145 \quad-0.8507558128 \quad 3.1813193360$

C $2.3533097934-1.4169998958 \quad 4.3372834475$

C $\quad 0.4759094214 \quad 2.9863314433 \quad 2.8580841606$

C $0.0343369476 \quad 4.1454795356 \quad 3.5454064325$

C $-1.2776638964 \quad 4.4704242634 \quad 3.5503432608$

C $-3.5909484794 \quad 3.9347783213 \quad 2.8051631702$

C $-4.4039844391 \quad 3.0962088649 \quad 2.1080946678$

C $-3.89906209531 .9629422295 \quad 1.4464235054$

C $-2.5653659000 \quad 1.6609534592 \quad 1.4824657830$

C $-1.7017900712 \quad 2.5277003974 \quad 2.2085273517$

C $-2.2083853471 \quad 3.6551213900 \quad 2.8616275424$

C $3.9014098124 \quad 3.6371585710-0.8141999911$

C $3.8332816229 \quad 5.0350922658-1.0561561175$

C $2.64071799395 .6363710484-1.2572441683$

C $\quad 0.1616884320 \quad 5.4247379098 \quad-1.3907270736$

C $-0.9267277416 \quad 4.6099737123 \quad-1.3083296813$

$\begin{array}{lllll}\text { C } & -0.7932864755 & 3.2414070885 & -1.0237873109\end{array}$

C $\quad 0.4347780745 \quad 2.6777687791 \quad-0.8164955342$

C $\quad 1.5808866470 \quad 3.5106949813 \quad-0.9334163834$

C $1.4461864095 \quad 4.8738754785-1.2009041595$

C $1.6917243198 \quad-0.0408999527 \quad-3.6017214351$

C $1.9099285737 \quad-0.7804840245 \quad-4.7910002755$

C $3.0342534606-1.5176539889-4.9425885653$

C $5.2239974605-2.2499005916-4.0088265937$ 

C $6.1128737930-2.1675769402 \quad-2.9797568283$
C $5.8531437874-1.3828295945-1.8461114095$
C $4.6858420271-0.6717711033-1.7159656577$
C $3.7409337043 \quad-0.7678123808 \quad-2.7828121852$
C $4.0116798787-1.5314980748-3.9190630815$
ZN - 2.5893906623 $-1.0430727073-0.0060575146$
O $-4.4150319481 \quad-0.4146860071 \quad-0.3488606560$
O $-0.6497329736-1.4275359875 \quad 0.3900784990$
N -2.6804142366 $-1.4000216412-2.1027765708$
N $-2.8175210924-2.8013122356 \quad 1.1080188334$
C $-1.8361517869-1.9723038788-2.9280368718$
C $-2.1086276342-2.0328580480-4.3176305589$
C $-3.2475128482-1.4990205350-4.8161114167$
C $-5.4101733394-0.3444401065-4.3768201931$
$\begin{array}{lllll}\text { C } & -6.2583493787 & 0.1794562851 & -3.4483837074\end{array}$
C $-5.9455490882 \quad 0.1716121597 \quad-2.0807210631$
C $-4.7647651845-0.3528839005-1.6161797898$
C $-3.8624484727-0.8879669106-2.5856741206$
C $-4.1859326508-0.8985566233-3.9431755606$
C $-3.9002014965 \quad-3.4112246856 \quad 1.5222904040$
C $-3.8265947778-4.7025230094 \quad 2.1093988049$
C $-2.6359416348 \quad-5.3261671997 \quad 2.2415650710$
C $-0.1655160034-5.2559833765 \quad 1.9247743573$
$\begin{array}{lllll}\text { C } & 0.9168179600 & -4.5498173157 & 1.4943965777\end{array}$
C $0.7797659799 \quad-3.2578830984 \quad 0.9616710682$
C $-0.4457522237 \quad-2.6609848350 \quad 0.8584009845$
C $-1.5867688474 \quad-3.4010281513 \quad 1.2721882589$
C $-1.4479017000-4.6789364714 \quad 1.8158801817$
H $-0.5519943327-1.61560234096 .0503891459$
H $1.8405149526-2.1236670567 \quad 6.3056042623$
H $4.1502548753 \quad-2.1285818583 \quad 5.2843493882$
H $5.5637983798-1.61192038513 .3423093492$
H $4.5807990044 \quad-0.6185114441 \quad 1.3041099896$
H $\quad 0.7582396494 \quad 4.7505293417 \quad 4.0507753997$
$\mathrm{H}-1.6282396481 \quad 5.3442022549 \quad 4.0645090156$
$\mathrm{H}-3.9751067348 \quad 4.8004982315 \quad 3.3077412839$
H $-5.4567616467 \quad 3.2942618700 \quad 2.0536895102$
H $-4.5421811640 \quad 1.3102661965 \quad 0.8985653367$
H $4.7476551834 \quad 5.5904166257 \quad-1.0833042842$
H $2.5798502930 \quad 6.6887854547-1.4553747296$
$\mathrm{H} \quad 0.0616737028 \quad 6.4718495324 \quad-1.5969394482$
H $-1.9099094274 \quad 5.0116627969-1.4530132725$
H $-1.65999076872 .6207434553-0.9464274060$
H $\quad 1.1672154272 \quad-0.7397268880 \quad-5.5598881289$
H $3.2042008446-2.0857542122 \quad-5.8365476384$
H $5.4265126666 \quad-2.8402113919-4.8801711373$ 


\begin{tabular}{|c|c|c|c|}
\hline & & & \\
\hline & & -13220207470 & \\
\hline & & & \\
\hline & -3.458245 & -1.534122 & -5 \\
\hline & & & \\
\hline & -7.1930664206 & 0.6030246162 & -3.7 \\
\hline & -6.6 & & \\
\hline & -4.7357649309 & -5.1656003692 & \\
\hline & -2.5 & -6.307316 & \\
\hline & -0.0622948506 & -6.237954 & \\
\hline & 1.8 & -4.9 & \\
\hline & 1.6423 & -2.712 & \\
\hline & 0.43 & 0.78 & -3.4 \\
\hline & 0.0 & & -2. \\
\hline & -0.2 & 0.502 & -4.2 \\
\hline & $0.6^{\prime}$ & & -3. \\
\hline & -5.2 & -2.75 & \\
\hline & -5.1 & -1.8 & \\
\hline & -5.6 & -2.54 & 2.3 \\
\hline & -5.9 & -3.437 & \\
\hline & 1.94 & 2.65 & 2.8 \\
\hline & 2.18 & 2.09 & 3.7 \\
\hline $\mathrm{H}$ & 2.53793 & 3.55 & 2.8 \\
\hline & 2.201967 & 2.0474 & 2.0 \\
\hline & $-0.5 c$ & -2.59 & -2.4 \\
\hline & 0.11 & -2.7978 & -3.2 \\
\hline & -0.7 & -3.535 & -1. \\
\hline & -0.10 & -1.9550 & -1.6 \\
\hline & 5.2 & & -0.5 \\
\hline 11 & 749403 & 3.38 & 0.3 \\
\hline & 5.93 & 3.30 & -1.3 \\
\hline $\mathrm{H}$ & 5.188 & $1.943828 \mathrm{C}$ & 355869 \\
\hline & -1.808 & -0.536 & 3.9 \\
\hline $\mathrm{H}$ & -2.39 & -1.2874139808 & 4.4600472 \\
\hline & -2.10 & -0.51312189 & \\
\hline & -2.0282051502 & 0.4316444243 & 4.376547 \\
\hline
\end{tabular}

\section{Meq2Zn Tetramer}

164

ZN - $1.3823885416 \quad 0.1137433545 \quad 0.8407190431$

ZN $-4.4524261739-0.5376869710 \quad 0.2779108900$

$\begin{array}{lllll}\mathrm{O} & -3.2248653078 & 1.0048275490 & 0.6630476390\end{array}$

O $\quad 0.4510475495 \quad-0.8116553194 \quad 0.8162233003$

$\begin{array}{lllll}\text { O } & -2.6495044950 & -1.3202275715 & 0.1308733763\end{array}$

$\begin{array}{lllll}\mathrm{O} & -5.9114469791 & 0.0238484556 & 1.4364889266\end{array}$

$\mathrm{N} \quad-1.2182594486 \quad 1.9372354006 \quad 2.1251910627$ 


\begin{tabular}{|c|c|c|c|}
\hline $\mathrm{N}$ & -1.3618416383 & -1.0178814930 & .7644518780 \\
\hline $\mathrm{N}$ & -4.9097495152 & -2.6912075054 & 0.2331398839 \\
\hline $\mathrm{N}$ & -5.6824573096 & 0.1809964411 & -1.2208480030 \\
\hline $\mathrm{C}$ & -0.2365735771 & 2.3295472943 & 2.8975444423 \\
\hline $\mathrm{C}$ & -0.2871816162 & 3.5755723306 & 3.5720708705 \\
\hline $\mathrm{C}$ & -1.3492861520 & 4.3951842135 & 3.4067260545 \\
\hline $\mathrm{C}$ & -3.5672385736 & 4.7903070008 & 2.3500068860 \\
\hline $\mathrm{C}$ & -4.5529246355 & 4.3110566787 & 1.5465829622 \\
\hline $\mathrm{C}$ & -4.4604143239 & 3.0374593826 & 0.9575427105 \\
\hline $\mathrm{C}$ & -3.3765282713 & 2.2326433970 & 1.1666550105 \\
\hline $\mathrm{C}$ & -2.3196192710 & 2.7382940806 & 1.9718368470 \\
\hline $\mathrm{C}$ & -2.4226086424 & 3.9944318051 & 2.5762372609 \\
\hline $\mathrm{C}$ & -2.2687100000 & -1.0699278040 & 3.7068563561 \\
\hline $\mathrm{C}$ & -2.1150630594 & -1.9399426678 & 4.8173789125 \\
\hline $\mathrm{C}$ & -1.0117469512 & -2.7128769018 & 4.9272198246 \\
\hline $\mathrm{C}$ & 1.1968434623 & -3.3886710853 & 4.00052 \\
\hline $\mathrm{C}$ & 2.1219450074 & -3.2272501101 & 3.0183068518 \\
\hline $\mathrm{C}$ & 1.9016610956 & -2.3505290062 & 1.9396436807 \\
\hline $\mathrm{C}$ & 0.7386979508 & -1.6385182037 & 1.8248197438 \\
\hline $\mathrm{C}$ & -0.2308976714 & -1.7832930984 & 2.8599346930 \\
\hline $\mathrm{C}$ & -0.0038602848 & -2.6467503735 & 3.9349327822 \\
\hline $\mathrm{C}$ & -6.0551526844 & -3.3253951078 & 0.3123229997 \\
\hline $\mathrm{C}$ & -6.1097346433 & -4.7384554476 & 0.2318865060 \\
\hline $\mathrm{C}$ & -4.9736733086 & -5.4576602402 & 0.0760856369 \\
\hline $\mathrm{C}$ & -2.4928403531 & -5.4674142848 & -0.1493675196 \\
\hline $\mathrm{C}$ & -1.3484513787 & -4.7348173899 & -0.2011941839 \\
\hline $\mathrm{C}$ & -1.3713314059 & -3.3317321475 & -0.1097142183 \\
\hline $\mathrm{C}$ & -2.5447354626 & -2.6492064719 & 0.0336881449 \\
\hline $\mathrm{C}$ & -3.7486216561 & -3.4016691642 & 0.0871805619 \\
\hline $\mathrm{C}$ & -3.7263704478 & -4.7962728296 & -0.0002232969 \\
\hline $\mathrm{C}$ & -5.5353156385 & 0.1952877882 & -2.5190315909 \\
\hline $\mathrm{C}$ & -6.4889779991 & 0.8354901611 & -3.3462795639 \\
\hline $\mathrm{C}$ & -7.5668346389 & 1.4394025748 & -2.7912778140 \\
\hline $\mathrm{C}$ & -8.8511915910 & 2.0168713625 & -0.7308303498 \\
\hline $\mathrm{C}$ & -8.9273534035 & 1.9305859940 & 0.6286383570 \\
\hline $\mathrm{C}$ & -7.9493969110 & 1.2697066367 & 1.3862774199 \\
\hline $\mathrm{C}$ & -6.8574583398 & 0.6775071579 & 0.7964205734 \\
\hline $\mathrm{C}$ & -6.7731317601 & 0.7710723706 & -0.6324656777 \\
\hline $\mathrm{C}$ & -7.7505145819 & 1.4238129235 & -1.3850669739 \\
\hline $\mathrm{ZN}$ & 1.3820332596 & -0.1143583485 & -0.8391570497 \\
\hline $\mathrm{ZN}$ & 4.4528979755 & 0.5332114145 & -0.2778359237 \\
\hline $\mathrm{O}$ & 3.2235735418 & -1.0074312268 & -0.6629998340 \\
\hline $\mathrm{O}$ & -0.4508181132 & 0.8123491919 & -0.8143478890 \\
\hline $\mathrm{O}$ & 2.6510250658 & 1.3182384464 & -0.1298276317 \\
\hline $\mathrm{O}$ & 5.9118535723 & -0.0351105095 & -1.4336880452 \\
\hline $\mathrm{N}$ & 1.2154762826 & -1.9361792472 & -2.1252671655 \\
\hline
\end{tabular}




\begin{tabular}{|c|c|c|c|}
\hline & & & \\
\hline & & & \\
\hline & 2 & -0 & \\
\hline & & & \\
\hline & & & \\
\hline & & & \\
\hline & & & \\
\hline & 769 & & \\
\hline & & & \\
\hline & & & \\
\hline & & & \\
\hline & & -3.9 & -2.5 \\
\hline & & & \\
\hline & 3 & & -4.8 \\
\hline & & & \\
\hline & & & \\
\hline & & & \\
\hline & & & \\
\hline & -0.7 & & \\
\hline & & & \\
\hline & 0.0 & & -3 \\
\hline & & & \\
\hline & 6.1 & & -0.2 \\
\hline & 4.9 & & \\
\hline & & 5.4 & \\
\hline & & & \\
\hline & & & \\
\hline & 2.5 & & \\
\hline & & & \\
\hline & 3.7 & & \\
\hline & & -0.1 & \\
\hline & 6.4 & -0.8 & \\
\hline & & -1.4 & \\
\hline & 8.8 & -2.0 & 0.7 \\
\hline & & & \\
\hline & & -1.2 & -1.3 \\
\hline & 6.8 & -0.6 & -0.7 \\
\hline & 6.7 & -0.7 & \\
\hline & 7.74 & -1.4 & \\
\hline & 0.5 & 3.8 & 4.1 \\
\hline & -1.39 & & \\
\hline & -3.640 & & \\
\hline & -5.4293418284 & 4.8 & 1.35 \\
\hline & -5.2623194494 & 2.68 & 0.35 \\
\hline & -2.8859734885 & 4180554 & 5.55 \\
\hline & -0.8813261945 & -3.3742 & \\
\hline
\end{tabular}




\begin{tabular}{|c|c|c|c|}
\hline $\mathrm{H}$ & 1.3598384943 & -4.0576355362 & 4.8222965581 \\
\hline & 3.0438294446 & -3.7745530920 & 3.0508692387 \\
\hline & 2.6495642739 & -2.2250582106 & 1.1931265209 \\
\hline $\mathrm{H}$ & -7.0626591030 & -5.2196607191 & 0.2987267603 \\
\hline & -5.0039511082 & -6.5281228385 & 0.0141654793 \\
\hline & -2.4780241272 & -6.5373824112 & -0.2138566916 \\
\hline & -0.3997221016 & -5.2209951645 & -0.3119419890 \\
\hline & -0.4614190816 & -2.7811615530 & -0.1535042682 \\
\hline & -6.3382835907 & 0.8340188021 & -4.4055030885 \\
\hline & -8.2952178004 & 1.9316049267 & -3.4059921384 \\
\hline$\Pi$ & -9.6033421409 & 2.5204597948 & -1.3045895466 \\
\hline & -9.7575330808 & 2.3767741709 & 1.1411672386 \\
\hline $\mathrm{H}$ & -8.0380962547 & 1.2190530625 & 2.4514757112 \\
\hline & -0.5419333965 & -3.8512011630 & -4.1955501933 \\
\hline & 1.3865612337 & -5.3455315505 & -3.9008021191 \\
\hline & 3.6322765974 & -5.7555435798 & -2.8173370687 \\
\hline $\mathrm{H}$ & 5.4224278979 & -4.9051909211 & -1.3622942566 \\
\hline $\mathrm{H}$ & 5.2585274878 & -2.6898313001 & -0.3507050364 \\
\hline & 2.8883527696 & 1.9701431640 & -5.5549469367 \\
\hline $\mathrm{H}$ & 0.8854542022 & 3.3813260532 & -5.7556026412 \\
\hline 1 & -1.3551560724 & 4.0659403574 & -4.8153237161 \\
\hline $\mathrm{H}$ & -3.0395719357 & 3.7821027099 & -3.0446086147 \\
\hline 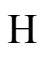 & -2.6473411404 & 2.2291730559 & -1.1892152244 \\
\hline & 7.0679398052 & 5.2122539903 & -0.3216358039 \\
\hline 1 & 5.0117752419 & 6.5235767238 & -0.0316416167 \\
\hline & 2.4869120838 & 6.5361271428 & 0.2059747521 \\
\hline $\mathrm{H}$ & 0.4072923431 & 5.2222372087 & 0.3141393179 \\
\hline & 0.4655102449 & 2.7822538724 & 0.1589571844 \\
\hline $\mathrm{H}$ & 6.3350376917 & -0.8210984847 & 4.4120491999 \\
\hline & 8.2918109378 & -1.9239327177 & 3.4180756891 \\
\hline $\mathrm{H}$ & 9.6003080211 & -2.5226043548 & 1.3201914830 \\
\hline & 9.7559768033 & -2.3896105401 & -1.1262682616 \\
\hline 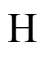 & 8.0379804063 & -1.2361049818 & -2.4423328610 \\
\hline O & -4.3182873700 & -0.4601357969 & -3.1143240984 \\
\hline $\mathrm{H}$ & -3.4687877629 & 0.2078079813 & -3.0316693570 \\
\hline 11 & -4.4744679232 & -0.6969273163 & -4.1584514936 \\
\hline $\mathrm{H}$ & -4.0835031826 & -1.3685055225 & -2.5769230169 \\
\hline $\mathrm{C}$ & 3.4779255096 & 0.1744167608 & -3.6550075104 \\
\hline & 3.7796698256 & -0.0429481325 & -2.6505828952 \\
\hline 11 & 4.3142886130 & 0.6258279217 & -4.1710450207 \\
\hline & 3.2447159279 & -0.7696487638 & -4.1389041516 \\
\hline . & 4.3163337773 & 0.4686031393 & 3.1146877159 \\
\hline & 4.0820189419 & 1.3749648391 & 2.5737339488 \\
\hline . & 4.4721484860 & 0.7090380029 & 4.1580334838 \\
\hline & 3.4666832405 & -0.1994148116 & 3.0342310458 \\
\hline & -3.4773853223 & -0.1729553727 & 3.6566744106 \\
\hline
\end{tabular}




$\begin{array}{cccc}\text { H } & -3.2426833208 & 0.7741384764 & 4.1338693828 \\ \text { H } & -4.3114470919 & -0.6213894424 & 4.1789169469 \\ \text { H } & -3.7832469421 & 0.0382538578 & 2.6521539268 \\ \text { C } & -0.9774261395 & -1.4535346905 & -3.0746561546 \\ \text { H } & -1.7877027541 & -2.0255721709 & -3.5045101815 \\ \text { H } & -0.7445019525 & -0.6300570542 & -3.7384469336 \\ \text { H } & -1.2967391102 & -1.0481083077 & -2.1289157563 \\ \text { C } & 7.3368315379 & 2.5453296452 & -0.5152426994 \\ \text { H } & 7.2294399273 & 1.8672813473 & -1.3461981368 \\ H & 7.5492620379 & 1.9536488920 & 0.3666086207 \\ \text { H } & 8.1670138280 & 3.2159469567 & -0.6885916102 \\ \mathrm{C} & -7.3350850137 & -2.5532504222 & 0.4968234901 \\ \mathrm{H} & -8.1661126906 & -3.2252078138 & 0.6606429670 \\ \mathrm{H} & -7.5423033818 & -1.9557652009 & -0.3822620422 \\ \mathrm{H} & -7.2330939980 & -1.8809126884 & 1.3331540399 \\ \mathrm{C} & 0.9748508415 & 1.4578199987 & 3.0756048887 \\ \mathrm{H} & 1.7851615308 & 2.0315256054 & 3.5031380057 \\ \mathrm{H} & 1.2935760458 & 1.0500420435 & 2.1306692452 \\ \mathrm{H} & 0.7430046359 & 0.6358243457 & 3.7416271023\end{array}$

\begin{tabular}{|c|c|c|c|}
\hline \multicolumn{4}{|c|}{$\begin{array}{l}\text { 4Meq2Zn Monomer } \\
\quad 41 \\
\text { scf done: }-2788.982656\end{array}$} \\
\hline $\mathrm{Zn}$ & -0.013762 & -0.003761 & 0.822942 \\
\hline $\mathrm{H}$ & & & \\
\hline $\mathrm{C}$ & 1.927488 & -1.4 & -1.0 \\
\hline $\mathrm{N}$ & & & \\
\hline $\mathrm{C}$ & 1.933599 & -3.8 & -1.2 \\
\hline $\mathrm{C}$ & & & \\
\hline $\mathrm{C}$ & 2.45 & -2. & -1. \\
\hline $\mathrm{C}$ & 0.8 & -3. & -0 . \\
\hline $\mathrm{C}$ & -0.6 & & \\
\hline $\mathrm{H}$ & 3.25 & -2.560605 & -2.2 \\
\hline $\mathrm{H}$ & 0.5 & & -0.2 \\
\hline $\mathrm{C}$ & -1.22 & -3.7 & 28 \\
\hline $\mathrm{H}$ & -2.0 & -3 & 393 \\
\hline $\mathrm{C}$ & -0.76 & & \\
\hline $\mathrm{H}$ & -1.2 & -5.8 & \\
\hline $\mathrm{C}$ & 0.24 & -5.072502 & 0.1 \\
\hline $\mathrm{H}$ & -2.30 & & -1.3 \\
\hline $\mathrm{C}$ & -1.90 & 1.457756 & -1.0 \\
\hline $\mathrm{N}$ & -0.941866 & & -0.1 \\
\hline $\mathrm{C}$ & -1.902011 & 3.848749 & 2209 \\
\hline $\mathrm{C}$ & -0.41 & & 57 \\
\hline $\mathrm{C}$ & -2.408123 & 2.639906 & -1.637658 \\
\hline
\end{tabular}




$\begin{array}{lrrl}\mathrm{C} & -0.855286 & 3.882842 & -0.290754 \\ \mathrm{C} & 0.625577 & 2.569662 & 1.205783 \\ \mathrm{H} & -3.196353 & 2.576540 & -2.358747 \\ \mathrm{H} & -0.574229 & 6.021417 & -0.184656 \\ \mathrm{C} & 1.172317 & 3.752870 & 1.633350 \\ \mathrm{H} & 1.944250 & 3.718194 & 2.372650 \\ \mathrm{C} & 0.731856 & 4.981804 & 1.123337 \\ \mathrm{H} & 1.189056 & 5.880185 & 1.489946 \\ \mathrm{C} & -0.255309 & 5.069943 & 0.184482 \\ \mathrm{O} & 0.975292 & 1.370803 & 1.627678 \\ \mathrm{O} & -1.026444 & -1.384190 & 1.587463 \\ \mathrm{C} & 2.485250 & -5.120633 & -1.821285 \\ \mathrm{H} & 3.287895 & -4.910566 & -2.515158 \\ \mathrm{H} & 1.707328 & -5.662430 & -2.347515 \\ \mathrm{H} & 2.869078 & -5.759847 & -1.034045 \\ \mathrm{C} & -2.436224 & 5.132906 & -1.853745 \\ \mathrm{H} & -1.642751 & 5.679078 & -2.351444 \\ \mathrm{H} & -2.844104 & 5.765874 & -1.073559 \\ \mathrm{H} & -3.217177 & 4.928125 & -2.573447\end{array}$

\section{Meq2Zn dimer}

82

$\begin{array}{llllll}\mathrm{ZN} & 30.0 & 20.7681140286 & 26.1978253363 & 9.8810899316\end{array}$

$\begin{array}{llllll}\mathrm{ZN} & 30.0 & 18.7826840854 & 27.7775999838 & 8.1200047747\end{array}$

$\begin{array}{llllll}\mathrm{O} & 8.0 & 18.7306979549 & 26.7743398610 & 9.7707722913\end{array}$

$\begin{array}{llllll}\mathrm{O} & 8.0 & 22.5883708045 & 25.5035482240 & 10.1681964837\end{array}$

$\begin{array}{llllll}\text { O } & 8.0 & 20.8198909332 & 27.2005237791 & 8.2298354072\end{array}$

$\begin{array}{lllll}\mathrm{O} & 8.0 & 16.9627439280 & 28.4737060555 & 7.8335662098\end{array}$

$\begin{array}{llllll}\mathrm{N} & \quad & 7.0 & 19.8448625619 & 24.4220968260 & 10.1254451591\end{array}$

$\mathrm{N} \quad \begin{array}{lllll}7.0 & 21.2047416563 & 27.3098254026 & 11.5155259055\end{array}$

$\begin{array}{lllll}\mathrm{N} & 7.0 & 19.7064556101 & 29.5535589973 & 7.8776225050\end{array}$

$\begin{array}{llllll}\mathrm{N} & \quad 7.0 & 18.3452348273 & 26.6681804479 & 6.4843185979\end{array}$

C $\quad \begin{array}{llllll}6.0 & 20.4686814528 & 23.2886904684 & 10.2899255326\end{array}$

C $\quad \begin{array}{llllll}6.0 & 19.7707157927 & 22.0971501436 & 10.5507715338\end{array}$

$\begin{array}{llllll}\text { C } & 6.0 & 18.4113776539 & 22.1031576600 & 10.6512807329\end{array}$

C $\quad \begin{array}{llllll}6.0 & 16.3116047073 & 23.4850237929 & 10.5592545003\end{array}$

$\begin{array}{llllll}\mathrm{C} & 6.0 & 15.7554009897 & 24.7129812957 & 10.3645601344\end{array}$

$\begin{array}{llllll}\mathrm{C} & 6.0 & 16.5388046881 & 25.8515630494 & 10.0975215607\end{array}$

$\begin{array}{lllll}\mathrm{C} & 6.0 & 17.8978290501 & 25.7566111803 & 10.0335618175\end{array}$

$\begin{array}{llllll}\text { C } & \quad 6.0 & 18.4879314680 & 24.4748397848 & 10.2201595528\end{array}$

$\begin{array}{llllll}\text { C } & 6.0 & 17.7172591414 & 23.3406648332 & 10.4834266983\end{array}$

$\begin{array}{lllll}\text { C } & 6.0 & 20.4567746733 & 28.1752294579 & 12.1400975712\end{array}$

$\begin{array}{llllll}\text { C } & 6.0 & 20.9317423963 & 28.8832172781 & 13.2562271371\end{array}$

C $\quad \begin{array}{llllll}6.0 & 22.1981603607 & 28.6722771765 & 13.7146672330\end{array}$

C $\quad \begin{array}{llllll}\text { C } & 24.3488820709 & 27.4043442532 & 13.4255745725\end{array}$

C $\quad \begin{array}{lllll}6.025 .0360301109 & 26.4687412622 & 12.7076864469\end{array}$ 


\begin{tabular}{|c|c|c|c|}
\hline $\mathrm{C}$ & $\begin{array}{ll}6.0 & 24.4767423947\end{array}$ & 25.8076579973 & 11 \\
\hline $\mathrm{C}$ & 6.023 .1955199166 & 26.0752065466 & 11.1861351610 \\
\hline $\mathrm{C}$ & 6.022 .4735870253 & 27.0608599080 & 11.9418897166 \\
\hline $\mathrm{C}$ & 6.023 .0247188412 & 27.7192725509 & 13.0432440737 \\
\hline $\mathrm{C}$ & $6.0 \quad 19.0830288580$ & 30.6873324922 & 7.7144633936 \\
\hline $\mathrm{C}$ & $6.0 \quad 19.7813804762$ & 31.8789339768 & 7.4547151040 \\
\hline $\mathrm{C}$ & $6.0 \quad 21.1406690922$ & 31.8725613139 & 7.3539614199 \\
\hline $\mathrm{C}$ & $6.0 \quad 23.2399954511$ & 30.4899311691 & 7.4446169942 \\
\hline $\mathrm{C}$ & $6.0 \quad 23.7958401005$ & 29.2616172238 & 7.6381991467 \\
\hline $\mathrm{C}$ & 6.023 .0120970409 & 28.1230418634 & 7.9041335391 \\
\hline $\mathrm{C}$ & 6.021 .6530840567 & 28.2183476650 & 7.9681204090 \\
\hline $\mathrm{C}$ & 6.021 .0633939893 & 29.5004537513 & 7.7828292222 \\
\hline $\mathrm{C}$ & 6.021 .8344125805 & 30.6346297947 & 7.5205760601 \\
\hline $\mathrm{C}$ & $\begin{array}{ll}6.0 & 19.0926753299\end{array}$ & 25.8027268389 & 5.8590987355 \\
\hline $\mathrm{C}$ & 6.018 .6180722049 & 25.0976616461 & 4.7410267410 \\
\hline $\mathrm{C}$ & $6.0 \quad 17.3525995369$ & 25.3115786531 & 4.2812606942 \\
\hline $\mathrm{C}$ & $6.0 \quad 15.2035362752$ & 26.5824470972 & 4.5699038949 \\
\hline $\mathrm{C}$ & $6.0 \quad 14.5168912410$ & 27.5175612298 & 5.2888618539 \\
\hline $\mathrm{C}$ & 6.015 .0757481416 & 28.1755707701 & 6.3941132671 \\
\hline $\mathrm{C}$ & $6.0 \quad 16.3559993910$ & 27.9052267144 & 6.8136517489 \\
\hline $\mathrm{C}$ & 6.017 .0774097375 & 26.9199767215 & 6.0567580076 \\
\hline $\mathrm{C}$ & 6.016 .5267212415 & 26.2646333009 & 4.9533581974 \\
\hline $\mathrm{H}$ & $1.0 \quad 21.5354234291$ & 23.3232004379 & 10.2291296147 \\
\hline $\mathrm{H}$ & $1.0 \quad 20.3230350600$ & 21.1888355240 & 10.6753493281 \\
\hline $\mathrm{H}$ & $1.0 \quad 15.6947580158$ & 22.6345227222 & 10.7600465648 \\
\hline $\mathrm{H}$ & $1.0 \quad 14.6900228551$ & 24.8245484373 & 10.4087689065 \\
\hline $\mathrm{H}$ & $1.0 \quad 16.0792900269$ & 26.8005624646 & 9.9164459680 \\
\hline $\mathrm{H}$ & $\begin{array}{ll}1.0 & 19.4659799540\end{array}$ & 28.3165488888 & 11.7623238508 \\
\hline $\mathrm{H}$ & $1.0 \quad 20.2863530357$ & 29.5873453736 & 13.7395848182 \\
\hline $\mathrm{H}$ & 1.024 .8009951296 & 27.8912545752 & 14.2635729440 \\
\hline $\mathrm{H}$ & $1.0 \quad 26.0413017905$ & 26.2243463011 & 12.9927339913 \\
\hline $\mathrm{H}$ & $1.0 \quad 25.0484484319$ & 25.0791288205 & 11.0680833091 \\
\hline $\mathrm{H}$ & 1.018 .0162941545 & 30.6531696929 & 7.7754612327 \\
\hline $\mathrm{H}$ & $1.0 \quad 19.2293325131$ & 32.7875789279 & 7.3313487926 \\
\hline $\mathrm{H}$ & $1.0 \quad 23.8571187764$ & 31.3404508341 & 7.2447545679 \\
\hline $\mathrm{H}$ & 1.024 .8611790956 & 29.1497596046 & 7.5937497713 \\
\hline $\mathrm{H}$ & $1.0 \quad 23.4713087905$ & 27.1737158005 & 8.0842290453 \\
\hline $\mathrm{H}$ & 1.020 .0828116141 & 25.6592922380 & 6.2378113267 \\
\hline $\mathrm{H}$ & 1.019 .2628612261 & 24.3932358973 & 4.2573000918 \\
\hline $\mathrm{H}$ & $1.0 \quad 14.7517484387$ & 26.0978511758 & 3.7303905284 \\
\hline $\mathrm{H}$ & $1.0 \quad 13.5123647441$ & 27.7641019870 & 5.0030324831 \\
\hline $\mathrm{H}$ & $1.0 \quad 14.5043468040$ & 28.9037704589 & 6.9313362574 \\
\hline $\mathrm{C}$ & 6.022 .7269911453 & 29.4260848865 & 14.9139739865 \\
\hline $\mathrm{H}$ & 1.023 .0063104680 & 28.7355698870 & 15.7021008113 \\
\hline $\mathrm{H}$ & $1.0 \quad 21.9784118338$ & 30.1044271062 & 15.3009936368 \\
\hline $\mathrm{H}$ & 1.023 .6053097500 & 30.0010361664 & 14.6420707864 \\
\hline
\end{tabular}




$\begin{array}{llllll}\mathrm{C} & & 6.0 & 17.6452906770 & 20.8313284753 & 10.9373104351 \\ \mathrm{H} & & 1.0 & 18.3207445320 & 19.9922732181 & 11.0354062728 \\ \mathrm{H} & & 1.0 & 16.9482398826 & 20.6197808290 & 10.1340405800 \\ \mathrm{H} & & 1.0 & 17.0812879678 & 20.9276838760 & 11.8584664863 \\ \mathrm{C} & & 6.0 & 21.9071968317 & 33.1443786814 & 7.0690628953 \\ \mathrm{H} & 1.0 & 22.4714982210 & 33.0485499705 & 6.1480453373 \\ \mathrm{H} & 1.0 & 22.6040663409 & 33.3550634998 & 7.8727358042 \\ \mathrm{H} & 1.0 & 21.2320402481 & 33.9837397816 & 6.9715570363 \\ \mathrm{C} & 6.0 & 16.8241814779 & 24.5608460793 & 3.0798423755 \\ \mathrm{H} & 1.0 & 17.5721331412 & 23.8819656498 & 2.6925787772 \\ \mathrm{H} & 1.0 & 16.5469479459 & 25.2532791435 & 2.2926600672 \\ \mathrm{H} & & 1.0 & 15.9445068092 & 23.9869018201 & 3.3495065628\end{array}$

\section{Meq2Zn trimer}

123

scf done: -8367.105180

$\begin{array}{rrrr}\mathrm{Zn} & 0.154849 & 0.328136 & 0.937812 \\ \mathrm{Zn} & 2.621627 & 0.352079 & -0.910404 \\ \mathrm{O} & 2.189662 & 0.010149 & 0.917616 \\ \mathrm{O} & -1.820933 & 0.771979 & 1.312915 \\ \mathrm{O} & 0.635498 & 1.057572 & -0.883009 \\ \mathrm{O} & 4.556596 & -0.024930 & -1.164178 \\ \mathrm{~N} & 0.539332 & -0.568423 & 2.857989 \\ \mathrm{~N} & 0.251011 & 2.240154 & 1.924892 \\ \mathrm{~N} & 2.859141 & 2.240431 & -1.626948 \\ \mathrm{~N} & 2.426802 & -1.275223 & -2.110743 \\ \mathrm{C} & -0.331503 & -0.821588 & 3.791398 \\ \mathrm{C} & 0.043500 & -1.381819 & 5.027311 \\ \mathrm{C} & 1.347309 & -1.683637 & 5.277545 \\ \mathrm{C} & 3.699651 & -1.689099 & 4.385210 \\ \mathrm{C} & 4.532530 & -1.403990 & 3.345302 \\ \mathrm{C} & 4.061848 & -0.832882 & 2.148229 \\ \mathrm{C} & 2.736575 & -0.544193 & 2.000296 \\ \mathrm{C} & 1.852605 & -0.851282 & 3.071028 \\ \mathrm{C} & 2.315728 & -1.415480 & 4.262262 \\ \mathrm{C} & 1.323620 & 2.918635 & 2.212828 \\ \mathrm{C} & 1.265756 & 4.147732 & 2.896844 \\ \mathrm{C} & 0.065627 & 4.668276 & 3.274164 \\ \mathrm{C} & -2.432115 & 4.366864 & 3.286433 \\ \mathrm{C} & -3.495952 & 3.594625 & 2.928309 \\ \mathrm{C} & -3.334707 & 2.370899 & 2.252114 \\ \mathrm{C} & -2.086061 & 1.920885 & 1.936107 \\ \mathrm{C} & -0.966893 & 2.724076 & 2.288942 \\ \mathrm{C} & -1.121914 & 3.937957 & 2.962975 \\ \mathrm{C} & 4.001978 & 2.760862 & -1.980925 \\ \mathrm{C} & 4.090341 & 4.074796 & -2.472838\end{array}$




$\begin{array}{cccc}\mathrm{C} & 2.971438 & 4.842554 & -2.593556 \\ \mathrm{C} & 0.481909 & 4.976442 & -2.273002 \\ \mathrm{C} & -0.654836 & 4.346818 & -1.864452 \\ \mathrm{C} & -0.644316 & 3.023829 & -1.388040 \\ \mathrm{C} & 0.522034 & 2.314098 & -1.318642 \\ \mathrm{C} & 1.716602 & 2.971420 & -1.732341 \\ \mathrm{C} & 1.713930 & 4.284400 & -2.208743 \\ \mathrm{C} & 1.339486 & -1.881603 & -2.499201 \\ \mathrm{C} & 1.381357 & -2.974532 & -3.380915 \\ \mathrm{C} & 2.574753 & -3.435946 & -3.848089 \\ \mathrm{C} & 5.075028 & -3.200922 & -3.800030 \\ \mathrm{C} & 6.151520 & -2.529338 & -3.297480 \\ \mathrm{C} & 6.015540 & -1.455824 & -2.406186 \\ \mathrm{C} & 4.778204 & -1.019870 & -1.996004 \\ \mathrm{C} & 3.645691 & -1.721736 & -2.530598 \\ \mathrm{C} & 3.776880 & -2.799364 & -3.409679 \\ \mathrm{Zn} & -2.723894 & -0.565823 & 0.293113 \\ \mathrm{O} & -4.677563 & -0.228025 & 0.430731 \\ \mathrm{O} & -0.753073 & -1.305264 & 0.172548 \\ \mathrm{~N} & -3.045277 & -0.034760 & -1.641209 \\ \mathrm{~N} & -2.967008 & -2.464606 & 0.979840 \\ \mathrm{C} & -2.181501 & 0.096149 & -2.609474 \\ \mathrm{C} & -2.583634 & 0.424240 & -3.914997 \\ \mathrm{C} & -3.900690 & 0.623979 & -4.199210 \\ \mathrm{C} & -6.245276 & 0.721614 & -3.303417 \\ \mathrm{H} & -7.059458 & 0.607444 & -2.214159 \\ \mathrm{H} & 2.260997 & 2.501590 & 1.908850 \\ \mathrm{C} & -6.566900 & 0.290575 & -0.940441 \\ \mathrm{C} & -5.226881 & 0.071771 & -0.726572 \\ \mathrm{C} & -4.371573 & 0.184605 & -1.874024 \\ \mathrm{C} & -4.856469 & 0.511594 & -3.142557 \\ \mathrm{C} & -4.105464 & -2.974326 & 1.362386 \\ \mathrm{C} & -4.199886 & -4.299265 & 1.822699 \\ \mathrm{C} & -3.092121 & -5.090254 & 1.878853 \\ \mathrm{C} & -0.615300 & -5.252324 & 1.482139 \\ \mathrm{C} & 0.522054 & -4.622993 & 1.074785 \\ \mathrm{C} & 0.516930 & -3.290367 & 0.625950 \\ \mathrm{C} & -0.644877 & -2.571217 & 0.581293 \\ \mathrm{C} & -1.836019 & -3.219723 & 1.018141 \\ \mathrm{H} & -1.3536375 & -4.543699 & 1.463103 \\ \mathrm{H} & -0.583989 & -1.568767 & 5.763480 \\ \mathrm{H} & -2.121118 & 5.285819 \\ \mathrm{H} & -1.666232 & 3.112035\end{array}$




\begin{tabular}{|c|c|c|c|}
\hline $\mathrm{H}$ & -2.581398 & 297 & \\
\hline & -4.489983 & 920817 & \\
\hline & -4.180185 & 780580 & 7 \\
\hline & 4.857678 & 74 & \\
\hline & 5.049236 & .460287 & O \\
\hline & 23 & & \\
\hline & -1.5 & 67317 & \\
\hline & -1.5 & 481 & -1 \\
\hline & 0.4 & 606 & -2 \\
\hline & 0.4 & 95 & -3.6 \\
\hline & 5.2 & 86 & -4 \\
\hline & 7.1 & -2.8 & -3. \\
\hline & & -0. & \\
\hline & -1.1 & -0 & -2. \\
\hline & & & -4 \\
\hline & -6.6 & 87 & -4.2 \\
\hline & & & -2 \\
\hline & -7.2 & 0.2 & -0 \\
\hline & -4.9 & -2.3 & \\
\hline & -5.1 & -4 & \\
\hline & -0.5 & -6. & \\
\hline & & & \\
\hline & 1.4 & -2.8 & 0.3 \\
\hline & 2.6 & -4.6 & -4.8 \\
\hline & & -4.9 & -5. \\
\hline & & & -4. \\
\hline & & -4 & -5 \\
\hline & -4.3 & 0.9 & -5.5 \\
\hline & $-5 .($ & 83 & -5 \\
\hline & -3.5 & 1.0 & -6.2 \\
\hline & -4.8 & 17 & -5.6 \\
\hline & -3.1 & -6.517 & 2.370451 \\
\hline & -4.1 & -6.7 & 2.6 \\
\hline & -2.5 & -6.657840 & 3.240 \\
\hline & & & \\
\hline & 3.0 & 6.2 & -3.1 \\
\hline & 4.0 & & -3.3 \\
\hline & 2.6 & & -2.3 \\
\hline & 2.4 & & -4.0 \\
\hline C & -0.0 & 5.989678 & 4.005092 \\
\hline & -0.4 & 5.860755 & 4.970881 \\
\hline & -0.6 & 6.703 & 3.4 \\
\hline & 0.96 & 6.402607 & 4.162759 \\
\hline & 1.77 & -2.291284 & 6.5 \\
\hline & 0.916707 & -2.422673 & 7.247585 \\
\hline & 2.488983 & -1.651714 & 7.0971 \\
\hline
\end{tabular}




\section{$\begin{array}{llll}H & 2.232795 & -3.259327 & 6.437095\end{array}$}

\begin{tabular}{|c|c|c|c|}
\hline \multicolumn{4}{|c|}{ 4Meq2Zn tetramer } \\
\hline $\mathrm{ZN}$ & -1.2897543688 & 0.5942276193 & -0.6597010443 \\
\hline $\mathrm{ZN}$ & -4.3846282579 & 0.2685142315 & -0.3148719545 \\
\hline $\mathrm{O}$ & -3.0558205552 & 0.2513340453 & -1.6886041581 \\
\hline $\mathrm{O}$ & 0.5040789511 & 1.1483995636 & 0.1507200330 \\
\hline $\mathrm{O}$ & -2.6969986042 & 0.8043656894 & 0.8177114042 \\
\hline $\mathrm{O}$ & -6.1076807371 & -0.0369748193 & -1.2679967699 \\
\hline $\mathrm{N}$ & -0.6648649716 & 0.4620082843 & -2.7227306501 \\
\hline $\mathrm{N}$ & -1.2382490925 & 2.7482293196 & -0.9815541303 \\
\hline $\mathrm{N}$ & -5.0400707684 & 1.9861652046 & 0.5366959062 \\
\hline $\mathrm{N}$ & -4.9058031917 & -1.4653943083 & 0.6073980518 \\
\hline $\mathrm{C}$ & 0.5521440289 & 0.5687187448 & -3.1713983078 \\
\hline $\mathrm{C}$ & 0.8365318342 & 0.5706227836 & -4.5513958866 \\
\hline $\mathrm{C}$ & -0.1684402089 & 0.4519247821 & -5.4616357866 \\
\hline $\mathrm{C}$ & -2.6438006485 & 0.1771505612 & -5.8176833033 \\
\hline $\mathrm{C}$ & -3.8767147889 & 0.0540008120 & -5.2510971836 \\
\hline $\mathrm{C}$ & -4.0621887957 & 0.0683676639 & -3.8562446550 \\
\hline $\mathrm{C}$ & -2.9906397965 & 0.2071344186 & -3.0219973194 \\
\hline $\mathrm{C}$ & -1.6968008029 & 0.3312665944 & -3.5990473195 \\
\hline $\mathrm{C}$ & -1.5086342302 & 0.3205882283 & -4.9839389090 \\
\hline $\mathrm{C}$ & -2.1273321823 & 3.4882730600 & -1.5783963630 \\
\hline $\mathrm{C}$ & -1.9815036367 & 4.8837124970 & -1.6897783514 \\
\hline $\mathrm{C}$ & -0.8958812859 & 5.5050809809 & -1.1529484676 \\
\hline $\mathrm{C}$ & 1.2502973184 & 5.2303149632 & 0.1260394834 \\
\hline $\mathrm{C}$ & 2.1123822358 & 4.3780855565 & 0.7478011182 \\
\hline $\mathrm{C}$ & 1.8913488820 & 2.9898830481 & 0.7855319481 \\
\hline $\mathrm{C}$ & 0.7898048068 & 2.4426038903 & 0.1877348037 \\
\hline $\mathrm{C}$ & -0.1326242278 & 3.3289727090 & -0.4441855824 \\
\hline $\mathrm{C}$ & 0.0863130907 & 4.7088042805 & -0.4891950613 \\
\hline $\mathrm{C}$ & -6.2159300810 & 2.5150953597 & 0.3399757754 \\
\hline $\mathrm{C}$ & -6.6195917571 & 3.6786078310 & 1.0167759917 \\
\hline $\mathrm{C}$ & -5.7827357986 & 4.2809579124 & 1.9076039804 \\
\hline $\mathrm{C}$ & -3.5303700028 & 4.2438489217 & 3.0195462075 \\
\hline $\mathrm{C}$ & -2.3234482882 & 3.6242058635 & 3.1380597377 \\
\hline $\mathrm{C}$ & -2.0067505801 & 2.4620186823 & 2.4129752974 \\
\hline $\mathrm{C}$ & -2.9098324834 & 1.9009775969 & 1.5539255238 \\
\hline $\mathrm{C}$ & -4.1709616759 & 2.5534556955 & 1.4156351458 \\
\hline $\mathrm{C}$ & -4.4930672547 & 3.7092383101 & 2.1322881240 \\
\hline $\mathrm{C}$ & -4.2744659349 & -2.1151522496 & 1.5430589778 \\
\hline $\mathrm{C}$ & -4.7783150567 & -3.3096778370 & 2.0834817554 \\
\hline $\mathrm{C}$ & -5.9553204086 & -3.8239776644 & 1.6314547516 \\
\hline $\mathrm{C}$ & -7.8911116126 & -3.5536963225 & 0.0550721194 \\
\hline $\mathrm{C}$ & -8.4760796130 & -2.7994807932 & -0.9205755962 \\
\hline
\end{tabular}



C $\quad-7.9026067772-1.6113667770-1.3934823652$
C $\quad-6.7094848627-1.1451912381-0.8942719458$
C $\quad-6.0914294313-1.9412972513 \quad 0.1276826709$
C $-6.6621238989-3.1237196811 \quad 0.6053691466$
ZN $\quad 1.2896068501 \quad-0.5936814341 \quad 0.6600531412$
$\begin{array}{lllll}\text { ZN } & 4.3846066862 & -0.2693376692 & 0.3143889286\end{array}$
O $3.0561455017 \quad-0.2518214684 \quad 1.6885383847$
$\begin{array}{llll}\text { O } & -0.5041749087 & -1.1478687043 & -0.1503873805\end{array}$
O $2.6966449121 \quad-0.8038420014-0.8176932505$
$\begin{array}{lllll}\mathrm{O} & 6.1080140179 & 0.0344854619 & 1.2673223777\end{array}$
N $\quad 0.6651565892 \quad-0.4605079495 \quad 2.7230694687$
N $1.2380429499 \quad-2.7475281162 \quad 0.9822661499$
N $5.0390078623 \quad-1.9871313680 \quad-0.5379478202$
N $4.9066370654 \quad 1.4648444128 \quad-0.6069675437$
C $\quad-0.5518653414 \quad-0.5662128462 \quad 3.1719397663$
C $\quad-0.8360616150 \quad-0.5674378334 \quad 4.5519793708$
C $\quad 0.1691252301-0.4491634644 \quad 5.4620388928$
C $\quad 2.6447101448-0.1759236147 \quad 5.8176526304$
C $3.8776418109 \quad-0.0538948639 \quad 5.2508585680$
C $\quad 4.0629008515-0.0687588372 \quad 3.8559857068$
C $\quad 2.9911640440-0.2071942095 \quad 3.0219187903$
C $\quad 1.6973110174 \quad-0.3302178462 \quad 3.5991949597$
C $\quad 1.5093395022-0.3188875664 \quad 4.9841083908$
C $\quad 2.1270924385-3.4874806868 \quad 1.5792786791$
C $\quad 1.9813332220-4.8829198676 \quad 1.6907181640$
C $\quad 0.8957781568 \quad-5.5043764574 \quad 1.1538489256$
C $\quad-1.2501783262 \quad-5.2298368312 \quad-0.1255572741$
C $-2.1121277383-4.3777131739-0.7476484704$
C $\quad-1.8912495581 \quad-2.9894835271 \quad-0.7852976597$
C $\quad-0.7898340119-2.4420994371 \quad-0.1873729640$
C $\quad 0.1325576179 \quad-3.3283788086 \quad 0.4447255432$
C $\quad-0.0863606474 \quad-4.7082072614 \quad 0.4898927028$
C $6.2146128759-2.5168385311-0.3418163278$
C $\quad 6.6173240302-3.6803865147-1.0191248125$
C $5.7798335496-4.2818827775-1.9099360086$
C $3.5270673654-4.2430581370-3.0210297828$
C $\quad 2.3204460834-3.6226956480-3.1388313066$
C $2.0047275351-2.4605271098-2.4132780977$
C $2.9084996758-1.9002993121 \quad-1.5544346222$
C $4.1692351371-2.5536538312-1.4167227853$
C $4.4904302497 \quad-3.7093153470 \quad-2.1339712347$
C $\quad 4.2755253608 \quad 2.1156245721 \quad-1.5420668479$
C $4.7802081192 \quad 3.3099830116-2.0820861470$
C $\quad 5.9576407157 \quad 3.8232329895 \quad-1.6299833930$
C $\quad 7.8935659236 \quad 3.5507240726-0.0541623988$
C $\quad 8.4782561122 \quad 2.7955260518 \quad 0.9208917277$ 


\begin{tabular}{|c|c|c|c|}
\hline $\mathrm{C}$ & 7.9040557984 & 1.6075568478 & 1.3932679859 \\
\hline $\mathrm{C}$ & 6.7104994650 & 1.1424820281 & 0.8940553443 \\
\hline C & 6.0927321962 & 1.9396265565 & -0.1272676079 \\
\hline $\mathrm{C}$ & 6.6641667131 & 3.1219045212 & -0.6044359172 \\
\hline $\mathrm{H}$ & 1.3340775205 & 0.6214961685 & -2.4438191898 \\
\hline & 1.8552326496 & 0.6530337340 & -4.8707937134 \\
\hline $\mathrm{H}$ & -2.5300432600 & 0.1640259292 & -6.8814654966 \\
\hline $\mathrm{H}$ & -4.7389744289 & -0.0570584302 & -5.8792598020 \\
\hline $\mathrm{H}$ & -5.0365181269 & -0.0254950568 & -3.4240977513 \\
\hline $\mathrm{H}$ & -2.9865110870 & 2.9955906701 & -1.9837956298 \\
\hline $\mathrm{H}$ & -2.7399058992 & 5.4470987826 & -2.1937397142 \\
\hline $\mathrm{H}$ & 1.4426670806 & 6.2825112035 & 0.1076842512 \\
\hline $\mathrm{H}$ & 2.9924825768 & 4.7663673137 & 1.2221710425 \\
\hline $\mathrm{H}$ & 2.5870426873 & 2.3439960595 & 1.2777099822 \\
\hline $\mathrm{H}$ & -6.8498602335 & 2.0098779731 & -0.3561374478 \\
\hline $\mathrm{H}$ & -7.5933595529 & 4.0797905522 & 0.8249251496 \\
\hline $\mathrm{H}$ & -3.7475949426 & 5.1275284244 & 3.5817382362 \\
\hline $\mathrm{H}$ & -1.5819413877 & 4.0294666721 & 3.7981342033 \\
\hline $\mathrm{H}$ & -1.0408982843 & 2.0184929280 & 2.5170937823 \\
\hline $\mathrm{H}$ & -3.3455470909 & -1.7084564316 & 1.8769505941 \\
\hline $\mathrm{H}$ & -4.2182120816 & -3.8113003351 & 2.8451529165 \\
\hline $\mathrm{H}$ & -8.3518896115 & -4.4552970722 & 0.3993609793 \\
\hline $\mathrm{H}$ & -9.4084442725 & -3.1207577442 & -1.3436693092 \\
\hline $\mathrm{H}$ & -8.3916964159 & -1.0453585386 & -2.1589296209 \\
\hline $\mathrm{H}$ & -1.3339442977 & -0.6187440238 & 2.4444931041 \\
\hline $\mathrm{H}$ & -1.8547775003 & -0.6490090968 & 4.8715450772 \\
\hline $\mathrm{H}$ & 2.5310955914 & -0.1623006504 & 6.8814435175 \\
\hline $\mathrm{H}$ & 4.7400555189 & 0.0568880326 & 5.8788578522 \\
\hline $\mathrm{H}$ & 5.0372357053 & 0.0242942902 & 3.4236811904 \\
\hline $\mathrm{H}$ & 2.9861559623 & -2.9947096362 & 1.9847963696 \\
\hline $\mathrm{H}$ & 2.7396405985 & -5.4462123747 & 2.1949253519 \\
\hline $\mathrm{H}$ & -1.4425130950 & -6.2820378556 & -0.1071366050 \\
\hline $\mathrm{H}$ & -2.9921671934 & -4.7660583524 & -1.2220800458 \\
\hline $\mathrm{H}$ & -2.5868916989 & -2.3436774355 & -1.2776457686 \\
\hline $\mathrm{H}$ & 6.8490099858 & -2.0123633127 & 0.3544069181 \\
\hline $\mathrm{H}$ & 7.5908839214 & -4.0822727869 & -0.8276886990 \\
\hline $\mathrm{H}$ & 3.7435410672 & -5.1266944697 & -3.5835818477 \\
\hline $\mathrm{H}$ & 1.5784167348 & -4.0273482529 & -3.7986903983 \\
\hline $\mathrm{H}$ & 1.0390903667 & -2.0164117908 & -2.5168283301 \\
\hline $\mathrm{H}$ & 3.3462252903 & 1.7098139214 & -1.8759753158 \\
\hline $\mathrm{H}$ & 4.2202555367 & 3.8124536979 & -2.8433094721 \\
\hline $\mathrm{H}$ & 8.3548985987 & 4.4521920851 & -0.3980565303 \\
\hline $\mathrm{H}$ & 9.4109541633 & 3.1159018067 & 1.3439350149 \\
\hline $\mathrm{H}$ & 8.3929249433 & 1.0407629773 & 2.1582739742 \\
\hline $\mathrm{C}$ & -6.5112858835 & -5.1113916291 & 2.1968109277 \\
\hline $\mathrm{H}$ & -5.8549290857 & -5.5092562552 & 2.9591903166 \\
\hline
\end{tabular}




$\begin{array}{lrrr}\mathrm{H} & -6.6185464038 & -5.8534544056 & 1.4133848072 \\ \mathrm{H} & -7.4877738175 & -4.9414796457 & 2.6368360255 \\ \mathrm{C} & -0.1163047146 & -0.4438899483 & 6.9475949914 \\ \mathrm{H} & 0.4017146327 & -1.2608947560 & 7.4379104767 \\ \mathrm{H} & -1.1769580069 & -0.5506118515 & 7.1323319248 \\ \mathrm{H} & 0.2177196950 & 0.4855782256 & 7.3955518698 \\ \mathrm{C} & 0.7275005465 & -7.0046872125 & 1.2482133043 \\ \mathrm{H} & 1.5560824161 & -7.4486003242 & 1.7839797707 \\ \mathrm{H} & 0.6842605080 & -7.4441925538 & 0.2577222909 \\ \mathrm{H} & -0.1907102275 & -7.2537901312 & 1.7688285209 \\ \mathrm{C} & -6.2024234824 & 5.5308289180 & 2.6484497482 \\ \mathrm{H} & -7.2027232563 & 5.8267116650 & 2.3620830257 \\ \mathrm{H} & -5.5251869065 & 6.3483989965 & 2.4273902115 \\ \mathrm{H} & -6.1879382704 & 5.3598116551 & 3.7191706831 \\ \mathrm{C} & -0.7275616331 & 7.0053946774 & -1.2471778738 \\ \mathrm{H} & 0.1904859768 & 7.2545105802 & -1.7680770155 \\ \mathrm{H} & -0.6839614311 & 7.4447800840 & -0.2566505556 \\ \mathrm{H} & -1.5563026817 & 7.4494154254 & -1.7826086235 \\ \mathrm{C} & 6.5143548306 & 5.1106126307 & -2.1946853170 \\ \mathrm{H} & 7.4907110532 & 4.9403639554 & -2.6348663901 \\ \mathrm{H} & 6.6221161786 & 5.8521626222 & -1.4108394790 \\ \mathrm{H} & 5.8581684058 & 5.5093119340 & -2.9567741078 \\ \mathrm{C} & 0.1172046064 & 0.4472678899 & -6.9471528801 \\ \mathrm{H} & 1.1778762920 & 0.5541567537 & -7.1316923002 \\ \mathrm{H} & -0.4008565266 & 1.2643850236 & -7.4372381908 \\ \mathrm{H} & -0.2166143348 & -0.4820811075 & -7.3955067670 \\ \mathrm{C} & 6.1985401736 & -5.5317105616 & -2.6514080768 \\ \mathrm{H} & 7.1987958182 & -5.8282411833 & -2.3655503499 \\ \mathrm{H} & 6.1837168386 & -5.3603071441 & -3.7220605913 \\ \mathrm{H} & 5.5209678614 & -6.3490018189 & -2.4303403685\end{array}$

\section{Meq2Zn monomer}

41 scf done: -2788.974691

$\begin{array}{crrr}\mathrm{Zn} & 0.008499 & 0.001429 & 0.832180 \\ \mathrm{H} & 2.331017 & -0.482551 & -1.316820 \\ \mathrm{C} & 1.930981 & -1.439077 & -1.050518 \\ \mathrm{~N} & 0.954784 & -1.465377 & -0.182243 \\ \mathrm{C} & 1.919682 & -3.813177 & -1.245241 \\ \mathrm{C} & 0.412405 & -2.655874 & 0.211156 \\ \mathrm{C} & 2.448332 & -2.614399 & -1.614620 \\ \mathrm{C} & 0.865198 & -3.877291 & -0.301561 \\ \mathrm{C} & -0.639697 & -2.572468 & 1.182684 \\ \mathrm{H} & 3.248684 & -2.552571 & -2.321520 \\ \mathrm{H} & 2.303508 & -4.719596 & -1.666017\end{array}$




$\begin{array}{rrrc}\mathrm{C} & -1.186804 & -3.760400 & 1.581795 \\ \mathrm{H} & -1.971765 & -3.741982 & 2.308021 \\ \mathrm{C} & -0.735790 & -4.987046 & 1.063784 \\ \mathrm{H} & -1.205268 & -5.884563 & 1.418331 \\ \mathrm{C} & 0.265150 & -5.086562 & 0.141521 \\ \mathrm{H} & -2.313092 & 0.476603 & -1.320686 \\ \mathrm{C} & -1.917397 & 1.434705 & -1.053593 \\ \mathrm{~N} & -0.941288 & 1.464499 & -0.185388 \\ \mathrm{C} & -1.917340 & 3.809027 & -1.245579 \\ \mathrm{C} & -0.404170 & 2.657081 & 0.209048 \\ \mathrm{C} & -2.440205 & 2.608203 & -1.616439 \\ \mathrm{C} & -0.863029 & 3.877001 & -0.301914 \\ \mathrm{C} & 0.648988 & 2.577565 & 1.179584 \\ \mathrm{H} & -3.240148 & 2.543451 & -2.323530 \\ \mathrm{H} & -2.305431 & 4.714159 & -1.665222 \\ \mathrm{C} & 1.190636 & 3.767564 & 1.579943 \\ \mathrm{H} & 1.976412 & 3.752083 & 2.305382 \\ \mathrm{C} & 0.733159 & 4.992723 & 1.064118 \\ \mathrm{H} & 1.198369 & 5.892126 & 1.419518 \\ \mathrm{C} & -0.268818 & 5.088550 & 0.142575 \\ \mathrm{O} & 1.011135 & 1.381113 & 1.605126 \\ \mathrm{O} & -0.995300 & -1.374856 & 1.610246 \\ \mathrm{C} & -0.739324 & 6.429115 & -0.387787 \\ \mathrm{H} & -0.167506 & 7.228905 & 0.065764 \\ \mathrm{H} & -1.786836 & 6.603034 & -0.159841 \\ \mathrm{H} & -0.609764 & 6.501190 & -1.463788 \\ \mathrm{C} & 0.729049 & -6.428633 & -0.390716 \\ \mathrm{H} & 0.153314 & -7.226298 & 0.061629 \\ \mathrm{H} & 1.775516 & -6.608134 & -0.162160 \\ \mathrm{H} & 0.600103 & -6.498495 & -1.466895\end{array}$

\section{Meq2Zn dimer}

82

ZN $1.1088052673 \quad 0.8983814026 \quad 0.5891153430$

ZN - $1.1069667061 \quad-0.8951154821 \quad-0.5896563703$

$\begin{array}{lllll}\text { O } & -0.3997641666 & 0.8756702369 & -0.8975022637\end{array}$

$\begin{array}{llll}\text { O } & 2.5326980161 & 1.1750343691 & 1.9159853494\end{array}$

$\begin{array}{llll}\text { O } & 0.4001044515 & -0.8718429320 & 0.8979829597\end{array}$

$\begin{array}{lllll}\text { O } & -2.5287235360 & -1.1726913719 & -1.9187763369\end{array}$

$\begin{array}{llll}\mathrm{N} & 0.1333931415 & 2.6193064083 & 0.9990816330\end{array}$

N $2.6598585175 \quad 0.9359685398 \quad-0.7176452350$

N $-0.1309713457-2.6164144346-0.9978540213$

$\begin{array}{llll}\mathrm{N} & -2.6596442488 & -0.9343362968 & 0.7148325306\end{array}$

$\begin{array}{llll}\text { C } & 0.4651485764 & 3.4264964496 & 1.9713423155\end{array}$

$\begin{array}{llll}\text { C } & -0.1930800335 & 4.6542019668 & 2.1548288104\end{array}$

C $-1.1855890275 \quad 5.0110894067 \quad 1.2944592001$ 

C $-2.5812045633 \quad 4.4453156762 \quad-0.7178996163$
C $-2.8339506444 \quad 3.5160779593 \quad-1.6810417009$
C $-2.12216528592 .3013699739-1.7799706926$
C $-1.1261018318 \quad 2.0054948674 \quad-0.9026931277$
C $-0.85144032692 .9525146707 \quad 0.1236485243$
C $-1.5553579680 \quad 4.1560371818 \quad 0.2262048148$
C $2.6278639829 \quad 0.8288658156-2.0187534294$
C $3.8084742000 \quad 0.8494717591 \quad-2.7781502006$
C $5.0037163757 \quad 0.9882983391 \quad-2.1408739850$
C $\quad 6.2637705183 \quad 1.2735405726 \quad 0.0120403046$
C $\quad 6.1497979012 \quad 1.3906433319 \quad 1.3663618542$
C $\quad 4.9169907502 \quad 1.3624023618 \quad 2.0437391014$
C $\quad 3.7331299805 \quad 1.2093003048 \quad 1.3724305305$
C $3.8370583064 \quad 1.0834960284 \quad-0.0548185899$
C $5.0613892103 \quad 1.1153369614 \quad-0.7302065150$
C $-0.4614913297-3.4239993005-1.9701871072$
C $0.1958759271 \quad-4.6525427956-2.1513887706$
C $1.1861033832-5.0098751644-1.2886035793$
C $2.5780265638-4.4439422245 \quad 0.7263074600$
C $2.8300052038 \quad-3.5140029514 \quad 1.6889703910$
$\begin{array}{llll}\text { C } & 2.1194093410 & -2.2984028561 & 1.7853455028\end{array}$
C $1.1252997093 \quad-2.0023658081 \quad 0.9059122524$
C $0.8517165793 \quad-2.9500103219 \quad-0.1201237540$
C $1.5545376270-4.1543665577 \quad-0.2202112156$
C $-2.6295976035 \quad-0.8266974953 \quad 2.0159072001$
C $-3.8112077386-0.8489079287 \quad 2.7737757975$
C $-5.0053616517-0.9897348633 \quad 2.1349414913$
C $-6.2621777211-1.2767073027-0.0196327534$
C $-6.1462307059-1.3938637448-1.3737618488$
C $-4.9125496969-1.3640403168-2.0495327985$
C $-3.7297993408-1.2090680430-1.3767324430$
C $-3.8358066351-1.08342717390 .0504004465$
C $-5.0609241383-1.1169091257 \quad 0.7241709421$
H $\quad 1.2675727652 \quad 3.1031059292 \quad 2.5989122054$
H $\quad 0.0979807410 \quad 5.2930851291 \quad 2.9619770779$
H $-3.6095330427 \quad 3.7037822012-2.3978362309$
H $-2.3763062740 \quad 1.5866646247 \quad-2.5346124471$
H $\quad \begin{array}{lllll}1.6625937011 & 0.7285517452 & -2.4679818373\end{array}$
H $3.7544395252 \quad 0.7572816085 \quad-3.8427000163$
H $7.0413702145 \quad 1.5119789032 \quad 1.9520865810$
H $4.8943909591 \quad 1.4607175431 \quad 3.1092950058$
H - $1.2623675481-3.1003920889-2.5996051741$
H $-0.0942127658-5.2916787502-2.9586773155$
H $3.6041286206 \quad-3.7018360074 \quad 2.4073207739$
H $2.3728463214 \quad-1.5832569050 \quad 2.5397983870$
H -1.6651694286 -0.7248099674 2.4665646559 


$\begin{array}{lrrr}\text { H } & -3.7587048917 & -0.7567595129 & 3.8384007006 \\ \text { H } & -7.0368197529 & -1.5164188682 & -1.9607535670 \\ \text { H } & -4.8886465108 & -1.4623663561 & -3.1150566847 \\ \text { H } & 1.6929082151 & -5.9449263199 & -1.4118311191 \\ \text { H } & 5.9127984034 & 1.0050563661 & -2.7064318113 \\ \text { H } & -1.6930882433 & 5.9455219532 & 1.4194791545 \\ \text { H } & -5.9151472913 & -1.0082284444 & 2.6992749905 \\ \text { C } & 3.3608803561 & -5.7402172319 & 0.6490934469 \\ \text { H } & 2.7097187869 & -6.6030086176 & 0.7516897262 \\ \text { H } & 4.0927463843 & -5.7813950356 & 1.4456952301 \\ \text { H } & 3.8924316683 & -5.8259724779 & -0.2937975476 \\ \text { C } & 7.6107452396 & 1.3107508565 & -0.6832657736 \\ \text { H } & 7.6741280361 & 2.1354181325 & -1.3875259914 \\ \text { H } & 7.8063257407 & 0.3893168399 & -1.2243381829 \\ \text { H } & 8.4020165800 & 1.4390399027 & 0.0450277214 \\ \text { C } & -7.6099956855 & -1.3147920811 & 0.6738127727 \\ \text { H } & -7.6741922200 & -2.1397844621 & 1.3776342225 \\ \text { H } & -8.4002418464 & -1.4432134956 & -0.0555916303 \\ \text { H } & -7.8069228599 & -0.3935814702 & 1.2147761567 \\ \text { C } & -3.3648334017 & 5.7409001969 & -0.6382731407 \\ \text { H } & -2.7145902783 & 6.6042743495 & -0.7418682967 \\ \text { H } & -3.8943936951 & 5.8258442120 & 0.3058127788 \\ \text { H } & -4.0985747610 & 5.7819606156 & -1.4331472347\end{array}$

\section{Meq2Zn trimer}

123

scf done: -8367.085669

$\begin{array}{cccc}\mathrm{Zn} & 2.125492 & -1.686156 & 0.674256 \\ \mathrm{O} & 3.999812 & -2.296231 & 0.897123 \\ \mathrm{O} & 0.039876 & -1.407603 & 0.566025 \\ \mathrm{~N} & 2.572811 & -1.907052 & -1.299690 \\ \mathrm{~N} & 1.457816 & -3.214920 & 1.846274 \\ \mathrm{C} & 1.827206 & -1.637289 & -2.338270 \\ \mathrm{C} & 2.272006 & -1.900734 & -3.644229 \\ \mathrm{C} & 3.507543 & -2.439102 & -3.828724 \\ \mathrm{C} & 5.660325 & -3.250198 & -2.826821 \\ \mathrm{C} & 6.353881 & -3.439251 & -1.667590 \\ \mathrm{C} & 5.832410 & -3.129953 & -0.398715 \\ \mathrm{C} & 4.571360 & -2.616803 & -0.246408 \\ \mathrm{C} & 3.825318 & -2.418867 & -1.456635 \\ \mathrm{C} & 4.347383 & -2.714974 & -2.720177 \\ \mathrm{C} & 2.238747 & -4.074741 & 2.445802 \\ \mathrm{C} & 1.711637 & -5.106994 & 3.240774 \\ \mathrm{C} & 0.363150 & -5.212012 & 3.387070 \\ \mathrm{C} & -1.922192 & -4.317305 & 2.853472 \\ \mathrm{C} & -2.620274 & -3.353899 & 2.190722\end{array}$




$\begin{array}{crrc}\mathrm{C} & -1.999759 & -2.356258 & 1.410805 \\ \mathrm{C} & -0.644026 & -2.308241 & 1.278903 \\ \mathrm{C} & 0.108097 & -3.298485 & 1.974306 \\ \mathrm{C} & -0.503275 & -4.290759 & 2.747070 \\ \mathrm{Zn} & 0.064948 & 0.595945 & 0.799315 \\ \mathrm{Zn} & -2.168364 & 1.297825 & -1.198215 \\ \mathrm{O} & -1.864635 & 1.294833 & 0.685276 \\ \mathrm{O} & 2.020495 & 0.135177 & 1.232616 \\ \mathrm{O} & -0.090169 & 0.949919 & -1.180858 \\ \mathrm{O} & -4.050731 & 1.832272 & -1.523335 \\ \mathrm{~N} & -0.608723 & 0.544343 & 2.853194 \\ \mathrm{~N} & 0.942776 & 2.518164 & 1.256856 \\ \mathrm{~N} & -1.501797 & 2.806374 & -2.396428 \\ \mathrm{~N} & -2.834495 & -0.486881 & -1.916634 \\ \mathrm{C} & 0.087102 & 0.165938 & 3.888968 \\ \mathrm{C} & -0.448829 & 0.198172 & 5.189592 \\ \mathrm{C} & -1.726310 & 0.628123 & 5.370557 \\ \mathrm{C} & -3.853660 & 1.496124 & 4.349404 \\ \mathrm{C} & -4.480550 & 1.848510 & 3.192412 \\ \mathrm{C} & -3.851062 & 1.791203 & 1.930608 \\ \mathrm{C} & -2.561261 & 1.373166 & 1.821927 \\ \mathrm{C} & -1.884495 & 0.982129 & 3.010022 \\ \mathrm{C} & -2.506129 & 1.041686 & 4.261511 \\ \mathrm{C} & 0.331226 & 3.669795 & 1.260068 \\ \mathrm{C} & 1.000733 & 4.859706 & 1.600135 \\ \mathrm{C} & 2.319894 & 4.808896 & 1.926998 \\ \mathrm{C} & 4.390518 & 3.418549 & 2.245001 \\ \mathrm{C} & 4.918927 & 2.164108 & 2.190621 \\ \mathrm{C} & 4.158699 & 1.025657 & 1.847611 \\ \mathrm{C} & 2.835910 & 1.142973 & 1.553296 \\ \mathrm{C} & 2.258222 & 2.442117 & 1.584906 \\ \mathrm{C} & 3.009786 & 3.570722 & 1.928019 \\ \mathrm{C} & -2.279878 & 3.688482 & -2.966626 \\ \mathrm{C} & -1.753926 & 4.704583 & -3.782805 \\ \mathrm{C} & -0.409737 & 4.768939 & -3.982657 \\ \mathrm{C} & 1.869434 & 3.821515 & -3.518203 \\ \mathrm{C} & 2.566702 & 2.855446 & -2.858530 \\ \mathrm{C} & 1.947031 & 1.870980 & -2.061358 \\ \mathrm{C} & 0.592815 & 1.837468 & -1.910902 \\ \mathrm{C} & -0.156735 & 2.846802 & -2.581295 \\ \mathrm{C} & 0.453519 & 3.823514 & -3.374506 \\ \mathrm{C} & -2.182606 & -1.614002 & -2.026756 \\ \mathrm{C} & -2.780522 & -2.750135 & -2.596147 \\ \mathrm{C} & -4.066758 & -2.675108 & -3.032690 \\ \mathrm{C} & -6.159789 & -1.317608 & -3.302577 \\ \mathrm{C} & -6.741221 & -0.102861 & -3.086396 \\ & & & \end{array}$




$\begin{array}{lrrr}\mathrm{C} & -6.067775 & 0.978596 & -2.490717 \\ \mathrm{C} & -4.761678 & 0.878042 & -2.089977 \\ \mathrm{C} & -4.132625 & -0.392047 & -2.317769 \\ \mathrm{C} & -4.805163 & -1.472231 & -2.898844 \\ \mathrm{H} & 0.883587 & -1.174663 & -2.148914 \\ \mathrm{H} & 1.637520 & -1.666321 & -4.473067 \\ \mathrm{H} & 7.348877 & -3.839349 & -1.718300 \\ \mathrm{H} & 6.430098 & -3.290424 & 0.474694 \\ \mathrm{H} & 3.286766 & -3.941890 & 2.291024 \\ \mathrm{H} & 2.376952 & -5.795644 & 3.718091 \\ \mathrm{H} & -3.690857 & -3.342961 & 2.259005 \\ \mathrm{H} & -2.601916 & -1.620273 & 0.921334 \\ \mathrm{H} & 1.086655 & -0.169050 & 3.707499 \\ \mathrm{H} & 0.152629 & -0.117686 & 6.016329 \\ \mathrm{H} & -5.498292 & 2.186188 & 3.231459 \\ \mathrm{H} & -4.380132 & 2.063673 & 1.040412 \\ \mathrm{H} & -0.704926 & 3.671051 & 0.994294 \\ \mathrm{H} & 0.465945 & 5.786525 & 1.594388 \\ \mathrm{H} & 5.958465 & 2.024746 & 2.417021 \\ \mathrm{H} & 4.611654 & 0.056449 & 1.802289 \\ \mathrm{H} & -3.324868 & 3.584324 & -2.774000 \\ \mathrm{H} & -2.416778 & 5.411659 & -4.235985 \\ \mathrm{H} & 3.636126 & 2.833391 & -2.940866 \\ \mathrm{H} & 2.549782 & 1.143712 & -1.559729 \\ \mathrm{H} & -1.191192 & -1.639663 & -1.630893 \\ \mathrm{H} & -2.219779 & -3.658236 & -2.670005 \\ \mathrm{H} & -7.764168 & 0.041539 & -3.378634 \\ \mathrm{H} & -6.582073 & 1.904701 & -2.336990 \\ \mathrm{H} & -0.052775 & -5.993545 & 3.989316 \\ \mathrm{H} & -4.535217 & -3.532470 & -3.470812 \\ \mathrm{H} & 3.859598 & -2.647185 & -4.818292 \\ \mathrm{H} & 0.005105 & 5.537354 & -4.602254 \\ \mathrm{H} & 2.845161 & 5.705605 & 2.184516 \\ \mathrm{H} & -2.150623 & 0.655400 & 6.353171 \\ \mathrm{C} & -4.558328 & 1.572770 & 5.689933 \\ \mathrm{H} & -4.046787 & 2.248598 & 6.368772 \\ \mathrm{H} & -4.615840 & 0.597226 & 6.163427 \\ \mathrm{H} & -5.569550 & 1.937221 & 5.559688 \\ \mathrm{H} & -2.316468 & -5.377705 & 3.682976 \\ \mathrm{H} & -5.329643 & 4.724615 \\ \mathrm{H} & -6.3762 & -5.376012 & 3.313554 \\ \mathrm{H} & -2.464640 & -3.940562 \\ \mathrm{H} & -2.318424 & -3.272454 \\ \mathrm{H} & -2.153802 & -4.182416\end{array}$




$\begin{array}{llll}\mathrm{C} & 6.257200 & -3.588727 & -4.179096 \\ \mathrm{H} & 7.256917 & -3.986470 & -4.055201 \\ \mathrm{H} & 6.327838 & -2.711288 & -4.815628 \\ \mathrm{H} & 5.666954 & -4.337014 & -4.700360 \\ \mathrm{C} & 2.565678 & 4.865178 & -4.369636 \\ \mathrm{H} & 3.635907 & 4.701746 & -4.357802 \\ \mathrm{H} & 2.379793 & 5.868653 & -3.998483 \\ \mathrm{H} & 2.235045 & 4.819641 & -5.403026 \\ \mathrm{C} & 5.235007 & 4.620692 & 2.620887 \\ \mathrm{H} & 6.252650 & 4.312601 & 2.825409 \\ \mathrm{H} & 4.851787 & 5.111898 & 3.510327 \\ \mathrm{H} & 5.264510 & 5.350704 & 1.817525\end{array}$

\begin{tabular}{|c|c|c|c|}
\hline \multicolumn{4}{|c|}{$5 \mathrm{Meq} 2 \mathrm{Zn}$ tetramer } \\
\hline $\mathrm{ZN}$ & -1.1709305410 & -0.6721169707 & 0.7870372930 \\
\hline $\mathrm{ZN}$ & -4.2353072934 & -1.1431344209 & 0.3837640713 \\
\hline $\mathrm{O}$ & -3.0171684859 & -0.4822080793 & 1.6970423491 \\
\hline $\mathrm{O}$ & 0.7403502550 & -1.0100769753 & 0.1540861336 \\
\hline $\mathrm{O}$ & -2.4146671545 & -1.5575419297 & -0.5794105373 \\
\hline $\mathrm{O}$ & -6.0312851986 & -1.0001297793 & 1.2273747863 \\
\hline $\mathrm{N}$ & -0.6993094739 & 0.1215506488 & 2.7497866733 \\
\hline $\mathrm{N}$ & -0.6798748443 & -2.6083774690 & 1.6783776789 \\
\hline $\mathrm{N}$ & -4.4582125744 & -3.1261617649 & 0.0110040818 \\
\hline $\mathrm{N}$ & -5.0653534424 & 0.1406362998 & -0.9605202628 \\
\hline $\mathrm{C}$ & 0.4913687209 & 0.4071855243 & 3.1982203181 \\
\hline $\mathrm{C}$ & 0.6958110739 & 0.8254215499 & 4.5272927950 \\
\hline $\mathrm{C}$ & -0.3727432535 & 0.9419228327 & 5.3599292842 \\
\hline $\mathrm{C}$ & -2.8593293520 & 0.7572085378 & 5.6832184733 \\
\hline $\mathrm{C}$ & -4.0472694846 & 0.4487573429 & 5.0921545154 \\
\hline $\mathrm{C}$ & -4.1510614782 & 0.0313254059 & 3.7482763669 \\
\hline $\mathrm{C}$ & -3.0386331664 & -0.0800235312 & 2.9722776785 \\
\hline $\mathrm{C}$ & -1.7833362973 & 0.2402336153 & 3.5589981463 \\
\hline $\mathrm{C}$ & -1.6793573164 & 0.6512860284 & 4.8923711853 \\
\hline $\mathrm{C}$ & -1.4326688042 & -3.3383103076 & 2.4540428815 \\
\hline $\mathrm{C}$ & -1.0041551832 & -4.5888655945 & 2.9348071219 \\
\hline $\mathrm{C}$ & 0.2197593013 & -5.0554213923 & 2.5696207057 \\
\hline $\mathrm{C}$ & 2.3414464070 & -4.7020706706 & 1.2728225039 \\
\hline $\mathrm{C}$ & 3.0225347840 & -3.8619630767 & 0.4450210236 \\
\hline $\mathrm{C}$ & 2.5164794391 & -2.6106777507 & 0.0362567489 \\
\hline $\mathrm{C}$ & 1.2971220098 & -2.1749362737 & 0.4628999074 \\
\hline $\mathrm{C}$ & 0.5507189613 & -3.0472054497 & 1.3105197772 \\
\hline $\mathrm{C}$ & 1.0550886195 & -4.2860176768 & 1.7220232793 \\
\hline $\mathrm{C}$ & -5.5074213597 & -3.8276081592 & 0.3500802169 \\
\hline $\mathrm{C}$ & -5.6153872239 & -5.1860195944 & 0.0087458839 \\
\hline
\end{tabular}



C $\quad-4.6091640691 \quad-5.7758623483-0.6923822921$
C $\quad-2.3622641527 \quad-5.5742782643 \quad-1.7927944150$
C $-1.3217368308-4.7399310476-2.0663671102$
C $-1.3011771282-3.3847517883-1.6775161834$
C $-2.3434476265-2.8283501515-0.9984600995$
C $-3.4388886226-3.6880436929-0.6882842754$
C $-3.4632165310-5.0335807918-1.0716876516$
C $\quad-4.5263072733 \quad 0.6514368238-2.0334394880$
C $\quad-5.2392045215 \quad 1.5285132425 \quad-2.8665220285$
C $\quad-6.5197916793 \quad 1.8533405831 \quad-2.5438286608$
C $\quad-8.4625243481 \quad 1.5999220581 \quad-0.9772132899$
C $\quad-8.9103439072 \quad 0.9996268025 \quad 0.1630639772$
C $\quad-8.1240677207 \quad 0.1240210896 \quad 0.9321131185$
C $-6.8362222396 \quad-0.1830506394 \quad 0.5788776516$
C $\quad-6.3477632804 \quad 0.4448283661 \quad-0.6156902965$
C $\quad-7.1310376475 \quad 1.3128364461-1.3842416054$
ZN $1.1705262565 \quad 0.6718170445 \quad-0.7879671019$
ZN $4.2347850029 \quad 1.1414921232 \quad-0.3842186863$
O $3.0167120009 \quad 0.4811741005-1.6977780180$
O $\quad-0.7406930304 \quad 1.0095969796-0.1557305222$
O $\quad 2.4141295585 \quad 1.5581292500 \quad 0.5778401768$
O $\quad 6.0307398370 \quad 0.9957679082 \quad-1.2274064749$
N $\quad 0.6986224861-0.1194174891 \quad-2.7514461326$
$\mathrm{N} \quad 0.6796246806 \quad 2.6086853251 \quad-1.6791929865$
$\mathrm{N} \quad 4.4595449464 \quad 3.1246892805 \quad-0.0127932893$
N $5.0634003164 \quad-0.1416760477 \quad 0.9615770462$
C $-0.4922523143 \quad-0.4034022280 \quad-3.2003197166$
C $\quad-0.6967964318 \quad-0.8211628026 \quad-4.5295646791$
C $\quad 0.3718715452 \quad-0.9390483228-5.3618261987$
C $2.8588554202-0.7575561966-5.6841396499$
C $4.0470016766-0.4507984412-5.0925827776$
C $4.1508700383-0.0336531108-3.7485979656$
C $\quad 3.0382838876 \quad 0.0790661144-2.9730183210$
C $\quad 1.7827929227 \quad-0.2394616075 \quad-3.5603127278$
C $1.6787447922-0.6502741047 \quad-4.8936946423$
C $\quad 1.4325947836 \quad 3.3391261681 \quad-2.4542432721$
C $\quad 1.0043078406 \quad 4.5901094906-2.9339826120$
C $\quad-0.2195538963 \quad 5.0565913500 \quad-2.5684257942$
C $\quad-2.3411953454 \quad 4.7027347187 \quad-1.2716958629$
C $\quad-3.0223604718 \quad 3.8621214530-0.4445140244$
C $\quad-2.5164782526 \quad 2.6104554787 \quad-0.0366168782$
C $\quad-1.2972904068 \quad 2.1747498168-0.4637393636$
C $\quad-0.5507872813 \quad 3.0474871778-1.3108677943$
C $\quad-1.0549668433 \quad 4.2867120000-1.7214166651$
C $\quad 5.5096908877 \quad 3.8248635014 \quad-0.3515198274$
C $\quad 5.6190232379 \quad 5.1832233093 \quad-0.0103508849$ 

C $\quad 4.6131421753 \quad 5.7742801289 \quad 0.6902306557$
C $\quad 2.3654976973 \quad 5.5754641172 \quad 1.7894531658$
C $\quad 1.3239969392 \quad 4.7422180150 \quad 2.0630174774$
C $\quad 1.3021886886 \quad 3.3870491156 \quad 1.6745618376$
$\begin{array}{llll}\text { C } & 2.3442143721 & 2.8292965939 & 0.9961097849\end{array}$
C $\quad 3.4405586604 \quad 3.6877024833 \quad 0.6860515330$
C $3.4661471177 \quad 5.0333850096 \quad 1.0691676891$
C $\quad 4.5238033844-0.6507011138 \quad 2.0350680800$
C $5.2359966031-1.5268847727 \quad 2.8696610866$
C $\quad 6.5165946084-1.8526254476 \quad 2.5479569450$
C $\quad 8.4600374437 \quad-1.6022659631 \quad 0.9817106273$
C $8.9085731280-1.0037147916-0.1591386319$
C $8.1229357454-0.1288202509 \quad-0.9297550231$
C $\quad 6.8350891016 \quad 0.1792059273 \quad-0.5775255919$
C $\quad 6.3458077708 \quad-0.4469554552 \quad 0.6176627524$
C $7.1284529989-1.3140390694 \quad 1.3878365809$
$\mathrm{H} \quad 1.2990573855 \quad 0.3427769253 \quad 2.5007944041$
H $\quad 1.6858616601 \quad 1.0591559977 \quad 4.8595856929$
H $\quad-4.9510867916 \quad 0.5226534821 \quad 5.6659470482$
H $\quad-5.1015387494 \quad-0.2067234301 \quad 3.3180390554$
H $\quad-2.3961521091 \quad-2.9502148307 \quad 2.7102161029$
H $\quad-1.6478143390 \quad-5.1581198522 \quad 3.5726950954$
H $3.9909971655 \quad-4.1522362562 \quad 0.0852974354$
H $3.0929422543 \quad-1.9860076992-0.6129731230$
H $\quad-6.2670889773 \quad-3.3052872995 \quad 0.8897155236$
H $\quad-6.4858485390 \quad-5.7357731298 \quad 0.2998236720$
$\mathrm{H} \quad-0.4706030372 \quad-5.1207910420 \quad-2.5968830894$
$\mathrm{H} \quad-0.4443592296-2.7879529460-1.9021081150$
H $\quad-3.5148355378 \quad 0.3835098409 \quad-2.2432406931$
H $\quad-4.7633978273 \quad 1.9351411777 \quad-3.7339829155$
$\mathrm{H} \quad-9.9116563318 \quad 1.1993202820 \quad 0.4949540686$
H $\quad-8.5320071600 \quad-0.3182007688 \quad 1.8174045569$
H $-1.3001160055-0.3380516683-2.5032257777$
H $-1.6870945970-1.0533533954-4.8622044753$
H $\quad 4.9509307351-0.5259234701 \quad-5.6660256361$
$\mathrm{H} \quad 5.1014616564 \quad 0.2032099265 \quad-3.3179710823$
H $\quad 2.3959833332 \quad 2.9510011936 \quad-2.7107097431$
H $\quad 1.6479317396 \quad 5.1596921293 \quad-3.5716263174$
H $\quad-3.9908144657 \quad 4.1522670229-0.0846521552$
$\mathrm{H} \quad-3.0929096467 \quad 1.9856017628 \quad 0.6124597459$
H $\quad 6.2689983025 \quad 3.3015322260-0.8907286692$
H $\quad 6.4900851655 \quad 5.7320952383 \quad-0.3012789607$
H $\quad 0.4731422915 \quad 5.1242774700 \quad 2.5931119501$
H $\quad 0.4447131247 \quad 2.7911276842 \quad 1.8989615810$
H $\quad 3.5123862732 \quad-0.3820304342 \quad 2.2442882053$
H $\quad 4.7597546061 \quad-1.9320833874 \quad 3.7375408719$ 


\begin{tabular}{|c|c|c|c|}
\hline & 00034704 & -1.2041199434 & -0.49030 \\
\hline $\mathrm{H}$ & 8.5316227558 & 0.3119915004 & -1.8154063825 \\
\hline & -4.6778573895 & -6.8097111164 & -0.9624282813 \\
\hline & 0.5580254427 & -6.0095794842 & 2.9182189809 \\
\hline & 7.0723481220 & -2.5223735364 & 3.1716471512 \\
\hline & -0.2339464296 & 1.2619234135 & 6.3722836597 \\
\hline & 4.6828886966 & 6.8080859981 & 0.9602005123 \\
\hline & -0.5575074092 & 6.0111717595 & -2.9161873263 \\
\hline & -7.0759847267 & 2.5238843457 & -3.1662438818 \\
\hline & 0.2331975247 & -1.2588748167 & -6.3742527845 \\
\hline & -9.3402839137 & 2.5367688660 & -1.7841826128 \\
\hline & -10.3126418833 & 2.6304331998 & -1.3164911451 \\
\hline & -9.4946251790 & 2.1689360720 & -2.7946417492 \\
\hline $\mathrm{H}$ & -8.9089387363 & 3.5316304456 & -1.8498451306 \\
\hline & 2.7831806688 & -1.2027286057 & -7.1318258185 \\
\hline $\mathrm{H}$ & 2.1741293235 & -0.5259730943 & -7.7237847994 \\
\hline & 2.3616583730 & -2.1997933456 & -7.2179286215 \\
\hline $\mathrm{H}$ & 3.7740605195 & -1.223 & -7.5679124031 \\
\hline C & -2.3531624992 & -7.0276790213 & -2.2245293065 \\
\hline $\mathrm{H}$ & -3.1843790266 & -7.2507455781 & -2.8868566484 \\
\hline $\mathrm{H}$ & -2.4119076372 & -7.6957917204 & -1.3704232422 \\
\hline $\mathrm{I}$ & -1.4372597722 & -7.2518911932 & -2.7565836100 \\
\hline $\mathrm{C}$ & 2.9161383166 & -6.0396691951 & 1.6973650883 \\
\hline & 2.2868044517 & -6.8629627855 & 1.3723970472 \\
\hline $\mathrm{H}$ & 3.0232918294 & -6.1028171367 & 2.7764353945 \\
\hline H & 3.8963013811 & -6.1799447746 & 1.2588556380 \\
\hline $\mathrm{C}$ & 9.3370712710 & -2.5383229156 & 1.7904579362 \\
\hline & 8.9058995601 & -3.5332377721 & 1.8562596932 \\
\hline $\mathrm{H}$ & 9.4901977844 & -2.1696070556 & 2.8007604851 \\
\hline $\mathrm{H}$ & 10.3100093921 & -2.6320016695 & 1.3239445364 \\
\hline C & -2.7836461104 & 1.2024186320 & 7.1308064335 \\
\hline & -3.7743023301 & 1.2219764462 & 7.5674753782 \\
\hline H & -2.3634103643 & 2.2000103094 & 7.2169557735 \\
\hline $\mathrm{H}$ & -2.1734169173 & 0.5264838672 & 7.7224968810 \\
\hline & 2.3579788211 & 7.0287709150 & 2.2215004086 \\
\hline $\mathrm{H}$ & 1.4421272602 & 7.2540637519 & 2.7531864685 \\
\hline & 2.4181348521 & 7.6970567973 & 1.3676175987 \\
\hline 11 & 3.1891861598 & 7.2505865684 & 2.8842639039 \\
\hline & -2.9158667077 & 6.0404164515 & -1.6956795693 \\
\hline 11 & -3.8963381238 & 6.1803578057 & -1.2577707916 \\
\hline & -3.0225915393 & 6.1040792073 & -2.7747802154 \\
\hline & -2.2867742671 & 6.8637376025 & -1.3703097923 \\
\hline
\end{tabular}


